\title{
Heterotic moduli stabilisation
}

\author{
M. Cicoli, ${ }^{a, b, c}$ S. de Alwis ${ }^{c, d}$ and A. Westphal ${ }^{e}$ \\ ${ }^{a}$ Dipartimento di Fisica ed Astronomia, Università di Bologna, \\ Bologna, Italy \\ ${ }^{b}$ INFN, Sezione di Bologna, \\ Bologna, Italy \\ ${ }^{c}$ Adbus Salam ICTP, \\ Strada Costiera 11, Trieste 34014, Italy \\ ${ }^{d}$ UCB 390 Physics Dept. University of Colorado, \\ Boulder CO 80309, U.S.A. \\ e Deutsches Elektronen-Synchrotron DESY, Theory Group, \\ D-22603 Hamburg, Germany \\ E-mail: mcicoli@ictp.it, dealwiss@colorado.edu, \\ alexander.westphal@desy.de
}

ABSTRACT: We perform a systematic analysis of moduli stabilisation for weakly coupled heterotic string theory compactified on internal manifolds which are smooth Calabi-Yau three-folds up to $\alpha^{\prime}$ effects. We first review how to stabilise all the geometric and gauge bundle moduli in a supersymmetric way by including fractional fluxes, the requirement of a holomorphic gauge bundle, D-terms, higher order perturbative contributions to the superpotential as well as non-perturbative and threshold effects. We then show that the inclusion of $\alpha^{\prime}$ corrections to the Kähler potential leads to new stable Minkowski (or de Sitter) vacua where the complex structure moduli and the dilaton are fixed supersymmetrically at leading order, while the stabilisation of the Kähler moduli at a lower scale leads to spontaneous breaking supersymmetry. The minimum lies at moderately large volumes of all the geometric moduli, at perturbative values of the string coupling and at the right phenomenological value of the GUT gauge coupling. We also provide a dynamical derivation of anisotropic compactifications with stabilised moduli which allow for perturbative gauge coupling unification around $10^{16} \mathrm{GeV}$. The value of the gravitino mass can be anywhere between the GUT and the TeV scale depending on the stabilisation of the complex structure moduli. In general, these are fixed by turning on background fluxes, leading to a gravitino mass around the GUT scale since the heterotic three-form flux does not contain enough freedom to tune the superpotential to small values. Moreover accommodating the observed value of the cosmological constant is a challenge. Low-energy supersymmetry 
could instead be obtained by focusing on particular Calabi-Yau constructions where the gauge bundle is holomorphic only at a point-like sub-locus of complex structure moduli space, or situations with a small number of complex structure moduli (like orbifold models), since in these cases one may fix all the moduli without turning on any quantised background flux. However obtaining the right value of the cosmological constant is even more of a challenge in these cases. Another option would be to focus on compactifications on non-complex manifolds, since these allow for new geometric fluxes which could be used to tune the superpotential as well as the cosmological constant, even if the moduli space of these manifolds is presently only poorly understood.

KEYwords: Flux compactifications, dS vacua in string theory, Superstrings and Heterotic Strings, Superstring Vacua

ArXiv ePRINT: 1304.1809 


\section{Contents}

1 Introduction $\quad 2$

2 Heterotic framework $\quad 7$

2.1 Tree-level expressions 8

$\begin{array}{lll}2.2 & \text { Corrections beyond leading order } & 10\end{array}$

2.2.1 Higher derivative effects 11

$\begin{array}{lll}2.2 .2 & \text { Loop effects } & 13\end{array}$

$\begin{array}{lll}2.2 .3 & \text { Non-perturbative effects } & 13\end{array}$

$\begin{array}{lll}2.3 & \text { Moduli-dependent Fayet-Iliopoulos terms } & 14\end{array}$

3 Supersymmetric vacua $\quad \mathbf{1 4}$

$\begin{array}{lll}3.1 & \text { Tree-level scalar potential } & 15\end{array}$

$\begin{array}{lll}\text { 3.1.1 Chern-Simons action and gauge bundle moduli } & 19\end{array}$

3.2 Corrections beyond tree-level 22

3.2.1 Step 1: $Z$ and $S$ stabilisation by gaugino condensation 23

3.2.2 Step 2: $T$ stabilisation by worldsheet instantons and threshold effects 24

$\begin{array}{ll}3.2 .3 & \text { Tuning the Calabi-Yau condition } \\ \end{array}$

$\begin{array}{lll}3.3 & \text { Flux vacua counting } & 26\end{array}$

4 Supersymmetry breaking vacua $\quad \mathbf{2 7}$

4.1 Fluxes, non-perturbative effects and threshold corrections 28

4.1.1 Derivation of the F-term potential 28

$\begin{array}{ll}\text { 4.1.2 Moduli stabilisation } & 30\end{array}$

4.2 Inclusion of $\alpha^{\prime}$ effects 31

4.3 Minkowski solutions 34

4.4 D-term potential 36

$\begin{array}{lll}\text { 4.4.1 } & \mathrm{D}+\text { F-term stabilisation } & 36\end{array}$

5 Moduli mass spectrum, supersymmetry breaking and soft terms $\quad 37$

$\begin{array}{lll}6 & \text { Anisotropic solutions } & 40\end{array}$

$\begin{array}{lll}7 & \text { Conclusions } & 41\end{array}$

A Dimensional reduction of 10D heterotic action 44 


\section{Introduction}

String theory is a candidate for a quantum theory of gravity with full unification of the forces of nature. As such it should be able to describe the patterns of the Standard Models (SMs) of particle physics and cosmology. For this description of 4D physics, string theory needs to compactify its ambient 10D space-time. The multitude of possible compactification choices together with a plethora of massless 4D 'moduli' fields originating from the deformation modes of the extra dimensions, leads to vacuum degeneracy and moduli problems. Recent progress in achieving moduli stabilisation points to the possibility of an exponentially large set of cosmologically distinct de Sitter (dS) solutions of string theory with positive but tiny cosmological constant, the 'landscape' (for reviews see [1, 2]).

These results need to be combined with string constructions of viable particle physics. One fruitful region of the string landscape for this purpose is weakly coupled heterotic string theory. Recent works on heterotic compactifications on both smooth Calabi-Yau (CY) manifolds [3] and their singular limits in moduli space, orbifolds [4-8], provided constructions of $4 \mathrm{D}$ low-energy effective field theories matching the minimal supersymmetric version of the SM (MSSM) almost perfectly. However, in contrast to the understanding achieved in type IIB string theory, heterotic CY or orbifold compactifications lack a well controlled description of moduli stabilisation, and consequently, of inflationary cosmology as well. ${ }^{1}$

As weakly coupled heterotic CY compactifications lack both D-branes and a part of the three-form flux available in type IIB, historically moduli stabilisation in the heterotic context focused mostly on the moduli dependence of 4D non-perturbative contributions to the effective action from gaugino condensation [11-13]. While this produced models of partial stabilisation of the dilaton and some Kähler moduli $[9,14-16]$, this route generically failed at describing controlled and explicit stabilisation of the $\mathcal{O}(100)$ complex structure moduli of a given CY. Moreover, the resulting vacua tend to yield values of the compactification radius and string coupling (given by the dilaton) at the boundary of validity of the supergravity approximation and the weak coupling regime.

The works [17-19] proposed to include the three-form flux $H$ to stabilise the complex structure moduli in combination with hidden sector gaugino condensation for supersymmetric dilaton stabilisation. The inclusion of fluxes in the heterotic string was originally studied by Strominger [20] who showed that, by demanding $\mathcal{N}=1$ supersymmetry, the classical 10D equations of motion imply $H=-\frac{i}{2}(\partial-\bar{\partial}) J$ where $J$ is the fundamental $(1,1)$-form on the internal space. Hence a non-vanishing three-form flux breaks the Kähler condition $d J=0$. Note that this is the case of $(0,2)$-compactifications which allow for MSSM-like model building and the generation of worldsheet instantons, since in the nonstandard embedding the Chern-Simons term gives a non-zero contribution to the three-form flux $H$. However this contribution is at order $\alpha^{\prime}$, implying that the Calabi-Yau condition is preserved at tree-level and broken only at order $\alpha^{\prime}$. Moreover, in the heterotic case, due to the absence of Ramond-Ramond three-form fluxes, there is generically no freedom to tune the superpotential small enough to fix the dilaton at weak coupling. However, a sufficiently small superpotential could be obtained by considering fractional Chern-Simons invariants

\footnotetext{
${ }^{1}$ However, for some recent attempts see e.g. [9, 10].
} 
(such as discrete Wilson lines) [17]. Note that it is natural to take these effects into account for $(0,2)$-compactifications which are the most relevant for both model building and moduli stabilisation since, as we pointed out above, they feature a non-vanishing Chern-Simons contribution to $H$, regardless of the presence of fractional Chern-Simons invariants. ${ }^{2}$

Supersymmetric vacua with all geometric moduli stabilised could be achieved by fixing the Kähler moduli via contributions from threshold corrections to the gauge kinetic function $[21,22]$. However this minimum cannot be trusted since it resides in a strong coupling regime where the gauge coupling is even driven into negative values [17]. The inclusion of a single worldsheet instanton contribution can resolve this difficulty [19]. However, none of these vacua break supersymmetry, resulting in unrealistic anti-de Sitter (AdS) solutions.

In this paper, we shall present new stable Minkowski (or de Sitter) vacua where all geometric moduli are stabilised and supersymmetry is broken spontaneously along the Kähler moduli directions. Let us summarise our main results:

- We identify two small parameters, one loop-suppressed and the other volumesuppressed, which allow us to expand the scalar potential in a leading and a subleading piece. This separation of scales allows us to perform moduli stabilisation in two steps.

- The leading scalar potential is generated by D-terms, quantised background fluxes (if needed for the stabilisation of the complex structure moduli), perturbative contributions to the superpotential and gaugino condensation. This potential depends on the gauge bundle moduli, the complex structure moduli and the dilaton which are all fixed supersymmetrically at leading order.

- The subleading scalar potential depends on the Kähler moduli and it is generated by threshold corrections to the gauge kinetic function, worldsheet instantons and $\mathcal{O}\left(\alpha^{\prime 2}\right)$ [23], and $\mathcal{O}\left(\alpha^{\prime 3}\right)[24,25]$ corrections to the Kähler potential. These effects give rise to new Minkowski vacua (assuming the fine-tuning problem can be solved) which break supersymmetry spontaneously along the Kähler moduli directions. The dilaton is stabilised at a value $\operatorname{Re}(\mathrm{S}) \simeq 2$ in a way compatible with gauge coupling unification, while the compactification volume is fixed at $\mathcal{V} \simeq 20$ which is the upper limit compatible with string perturbativity. These new minima represent a heterotic version of the type IIB LARGE Volume Scenario (LVS) [26, 27].

- By focusing on CY manifolds with K3- or $T^{4}$-fibres over a $\mathbb{P}^{1}$ base, we shall also show that this LVS-like moduli stabilisation mechanism allows for anisotropic constructions where the overall volume is controlled by two larger extra dimensions while the remaining four extra dimensions remain smaller. This anisotropic setup is particularly interesting phenomenologically, as it allows one to match the effective string scale to the GUT scale of gauge coupling unification [28, 29], and fits very well

\footnotetext{
${ }^{2}$ As we shall describe in section 3.1.1, the co-exact piece of the Chern-Simons term is responsible for the breaking of the Kähler condition $d J=0$ while the generation of fractional invariants is controlled by the harmonic piece of the Chern-Simons term.
} 
with the picture of intermediate 6D orbifold GUTs emerging from heterotic MSSM orbifolds $[29,30]$.

- The soft terms generated by gravity mediation feature universal scalar masses, Aterms and $\mu / B \mu$-term of order the gravitino mass, $m_{3 / 2}=|W| M_{P} / \sqrt{2 \operatorname{Re}(\mathrm{S}) \mathcal{V}}$, and suppressed gaugino masses at the \%-level. In turn, the value of the supersymmetry breaking scale depends on the stabilisation of the complex structure moduli:

1. If the complex structure moduli are fixed by turning on quantised background fluxes, due to the lack of tuning freedom in the heterotic three-form flux, $|W|$ can at most be made of order $|W| \simeq \mathcal{O}(0.1-0.01)$ by turning on only Chern-Simons fractional fluxes. Hence the gravitino mass becomes of order $M_{G U T} \simeq 10^{16} \mathrm{GeV}$ for $\operatorname{Re}(\mathrm{S}) \simeq 2$ and $\mathcal{V} \simeq 20$, leading to high scale supersymmetry breaking.

2. If the complex structure moduli are fixed without turning on quantised background fluxes, the main contribution to $|W|$ can come from higher order perturbative operators or gaugino condensation. Hence $|W|$ can acquire an exponentially small value, leading to low-scale supersymmetry [31, 32].

Let us discuss the stabilisation of the complex structure moduli in more detail. In a series of recent papers [33-35], it has been shown that in particular examples one could be able to fix all the complex structure moduli without the need to turn on any quantised background flux. Note that, as we explained above, if one focuses on $(0,2)$-compactifications, this observation is not important for preserving the CY condition (since this is broken at order $\alpha^{\prime}$ regardless of the presence of a harmonic quantised flux) but it is instead crucial to understand the order of magnitude of the superpotential which sets the gravitino mass scale once supersymmetry is broken. Following the original observation of Witten [36], the authors of [33-35] proved that, once the gauge bundle is required to satisfy the Hermitian Yang-Mills equations, the combined space of gauge bundle and complex structure moduli is not a simple direct product but acquires a 'cross-structure'. Denoting the gauge bundle moduli as $C_{i}, i=1, \ldots, N$, and the complex structure moduli as $Z_{\alpha}, \alpha=1, \ldots, h^{1,2}$, this observation implies that the dimensionality of the gauge bundle moduli space is actually a function of the complex structure moduli, i.e. $N=N(Z)$, and viceversa the number of massless $Z$-fields actually depends on the value of the gauge bundle moduli. As a simple intuitive example, consider a case with just one gauge bundle modulus and a leading order scalar potential which looks like:

$$
V=\left(\sum_{\beta=1}^{h_{\mathrm{fix}}^{1,2}}\left|Z_{\beta}\right|^{2}\right)|C|^{2} .
$$

The form of this potential implies that:

- If $C$ is fixed by some stabilisation mechanism (like D-terms combined with higher order $C$-dependent terms in the superpotential) at $\langle C\rangle \neq 0$, then $h_{\mathrm{fix}}^{1,2}$ complex structure moduli are fixed at $\left\langle Z_{\beta}\right\rangle=0 \forall \beta=1, \ldots, h_{\text {fix }}^{1,2}$. Hence the number of $Z$-moduli 
left flat is given by $h_{\text {hol }}^{1,2}=h^{1,2}-h_{\text {fix }}^{1,2}$, which is also the dimensionality of the sub-locus in complex structure moduli space for $C \neq 0$ where the gauge bundle is holomorphic. Hence the best case scenario is when this sub-locus is just a point, i.e. $h_{\text {hol }}^{1,2}=0$.

- If the $Z$-moduli are fixed by some stabilisation mechanism (like by turning on background quantised fluxes) at values different from zero, then the gauge bundle modulus $C$ is fixed at $\langle C\rangle=0 .^{3}$

However this stabilisation mechanism generically does not lead to the fixing of all complex structure moduli due to the difficulty of finding examples with $h_{\text {hol }}^{1,2}=0$, i.e. with a point-like sub-locus in complex structure moduli space where the gauge bundle is holomorphic. In fact, there is so far no explicit example in the literature where $h_{\text {hol }}^{1,2}=0$ can be obtained without having a singular CY even if there has been recently some progress in understanding how to resolve these singular point-like sub-loci [39]. Moreover, let us stress that even if one finds a non-singular CY example with $h_{\text {hol }}^{1,2}=0$ (there is in principle no obstruction to the existence of this best case scenario), all the complex structure moduli are fixed only if $C \neq 0$, since for $C=0$ the $Z$-directions would still be flat. As we pointed out above, $C \neq 0$ could be guaranteed by the interplay of D-terms and higher order terms in the superpotential, but in the case when the number of $C$-moduli is large, one should carefully check that all of them are fixed at non-zero values (for example, one might like to have some of them to be fixed at zero in order to preserve some symmetries relevant for phenomenology like $\left.\mathrm{U}(1)_{B-L}\right)$. Thus the requirement of a holomorphic gauge bundle generically fixes some complex structure moduli but not all of them. Note also that these solutions are not guaranteed to survive for a non-vanishing superpotential, since one would then need to solve a set of non-holomorphic equations.

Let us therefore analyse the general case where some $Z$-moduli are left flat after the requirement of a holomorphic gauge bundle, and summarise our results for their stabilisation:

- Given that promising phenomenological model building requires us to focus on the non-standard embedding where the $H$-flux already gets a non-vanishing contribution from the co-exact piece of the Chern-Simons term, we consider quite natural the option to turn on also a harmonic Chern-Simons piece that could yield a fractional $Z$-dependent superpotential that lifts the remaining complex structure moduli [17].

- If $H \neq 0$, as in the case of $(0,2)$-compactifications, both the dilaton and the warp factor could depend on the internal coordinates. For simplicity, we shall however restrict to the solutions where both of them are constant, corresponding to the case of 'special Hermitian manifolds' [40].

- The inclusion of quantised background fluxes cannot fix the remaining $h_{\text {hol }}^{1,2}>0$ complex structure moduli in a supersymmetric way with, at the same time, a vanishing flux superpotential $W_{0}$. In fact, setting the F-terms of the $Z$-moduli to zero corresponds to setting the $(1,2)$-component of $H$ to zero, whereas setting $W_{0}=0$ implies

\footnotetext{
${ }^{3}$ See also $[37,38]$ for a mathematical discussion of this issue which basically comes to the same conclusion that gauge bundle moduli are generically absent.
} 
a vanishing $(3,0)$-component of $H$. As a consequence, given that the flux is real, the entire harmonic flux $H$ is zero, and so the $h_{\text {hol }}^{1,2}>0 Z$-moduli are still flat. ${ }^{4}$ Note that this would not be the case in type IIB where the three-form flux is complex (because of the presence of also Ramond-Ramond fluxes) [41].

- The remaining $h_{\text {hol }}^{1,2}>0 Z$-moduli can be fixed only if $W_{0} \neq 0$ but this would lead to a runaway for the dilaton if $W_{0}$ is not fine-tuned to exponentially small values to balance the dilaton-dependent contribution from gaugino condensation. However, due to the absence of Ramond-Ramond fluxes, the heterotic $H$-flux does not contain enough freedom to tune $W_{0}$ to small values, since it is used mostly to stabilise the complex structure moduli in a controlled vacuum. There are then two options:

1. Models with either accidentally cancelling integer flux quanta or only ChernSimons fractional fluxes where the flux superpotential could be small enough to compete with gaugino condensation, even if this case would lead to supersymmetry breaking around the GUT scale;

2. Compactifications on non-Kähler manifolds which do not admit a closed holomorphic (3,0)-form, since these cases allow for new geometric fluxes which could play a similar rôle as type IIB Ramond-Ramond fluxes, and could be used to tune $W_{0}$ to small values $[40,42-45]$. In this case one could lower the gravitino mass to the $\mathrm{TeV}$ scale and have enough freedom to tune the cosmological constant. However, the moduli space of these manifolds is at present only poorly understood.

In this paper, we shall not consider the second option given that we want to focus on cases, like 'special Hermitian manifolds', which represent the smallest departure from a CY due to $\alpha^{\prime}$ effects.

This analysis suggests that if one is interested in deriving vacua where our Kähler moduli stabilisation mechanism leads to spontaneous supersymmetry breaking around the $\mathrm{TeV}$ scale, one should focus on one of the two following situations:

1. Models where the requirement of a holomorphic gauge bundle fixes all complex structure moduli without inducing singularities (so that the supergravity approximation is reliable), i.e. models with $h_{\mathrm{hol}}^{1,2}=0$ [33-35]. The dilaton could then be fixed in a supersymmetric way by using a double gaugino condensate while the Kähler moduli could be fixed following our LVS-like method by including worldsheet instantons, threshold and $\alpha^{\prime}$ effects. This global minimum would break supersymmetry spontaneously along the Kähler moduli directions. The gravitino mass could then be around the $\mathrm{TeV}$ scale because of the exponential suppression from gaugino condensation.

2. Simple models with a very small number of complex structure moduli, like Abelian orbifolds with a few untwisted $Z$-moduli, or even non-Abelian orbifolds with no complex structure moduli at all. In fact, in this case gauge singlets could be fixed at

\footnotetext{
${ }^{4}$ This statement is also implicit in [18].
} 
non-zero values via D-terms induced by anomalous $\mathrm{U}(1)$ factors and higher order terms in the superpotential [4-8], so resulting in cases where all the $Z$-moduli become massive by the holomorphicity of the gauge bundle. The dilaton could then be fixed by balancing gaugino condensation with the contribution from a gauge bundle modulus (i.e. a continuous Wilson line in the orbifold language) which develops a small vacuum expectation value (VEV) because it comes from $R$-symmetry breaking higher order terms in the superpotential $[31,32]$. A low gravitino mass could then be obtained due to this small VEV.

Let us finally note that accommodating our observed cosmological constant, which is a challenge even with fluxes and $\mathcal{O}(100)$ complex structures, is even more of a challenge in cases without quantised fluxes.

This paper is organised as follows. In section 2 we introduce the general framework of heterotic CY compactifications [46, 47], reviewing the form of the tree-level effective action and then presenting a systematic discussion of quantum corrections from non-perturbative effects [11-13], string loops [48-50], and higher-derivative $\alpha^{\prime}$-corrections [23-25] according to their successive level of suppression by powers of the string coupling and inverse powers of the volume. Supersymmetric vacua are then discussed in section 3, while in section 4 we derive new global minima with spontaneous supersymmetry breaking which can even be Minkowski (or slightly de Sitter) if enough tuning freedom is available. After discussing in section 5 the resulting pattern of moduli and soft masses generated by gravity mediation, we derive anisotropic constructions in section 6 . We finally present our conclusions in section 7 .

\section{Heterotic framework}

Let us focus on weakly coupled heterotic string theory compactified on a smooth CY threefold $X$. The 4 D effective supergravity theory involves several moduli: $h^{1,2}(X)$ complex structure moduli $Z_{\alpha}, \alpha=1, \ldots, h^{1,2}(X)$; the dilaton $S$ and $h^{1,1}$ Kähler moduli $T_{i}, i=$ $1, \ldots, h^{1,1}(X)$ (besides several gauge bundle moduli).

The real part of $S$ is set by the $4 \mathrm{D}$ dilaton (see appendix A for the correct normalisation):

$$
\operatorname{Re}(S) \equiv s=\frac{1}{4 \pi} e^{-2 \phi_{4}}=\frac{1}{4 \pi} e^{-2 \phi} \mathcal{V},
$$

where $\phi$ is the 10D dilaton whose VEV gives the string coupling $e^{\langle\phi\rangle}=g_{s}$. The imaginary part of $S$ is given by the universal axion $a$ which is the $4 \mathrm{D}$ dual of $B_{2}$. On the other hand, the real part of the Kähler moduli, $t_{i}=\operatorname{Re}\left(T_{i}\right)$, measures the volume of internal two-cycles in units of the string length $\ell_{s}=2 \pi \sqrt{\alpha^{\prime}}$. The imaginary part of $T_{i}$ is given by the reduction of $B_{2}$ along the basis $(1,1)$-form $\hat{D}_{i}$ dual to the divisor $D_{i}$.

We shall focus on general non-standard embeddings with possible $U(1)$ factors in the visible sector. Hence the gauge bundle in the visible $E_{8}^{\text {vis }}$ takes the form $V_{\text {vis }}=U_{\text {vis }} \bigoplus_{\kappa} \mathcal{L}_{\kappa}$ where $U_{\text {vis }}$ is a non-Abelian bundle whereas the $\mathcal{L}_{\kappa}$ are line bundles. On the other hand the vector bundle in the hidden $E_{8}^{\text {hid }}$ involves just a non-Abelian factor $V_{\text {hid }}=U_{\text {hid }}$. We shall not allow line bundles in the hidden sector since, just for simplicity, we shall not consider matter fields charged under anomalous $\mathrm{U}(1) \mathrm{s}$. In fact, if we want to generate a 
superpotential from gaugino condensation in the hidden sector in order to fix the moduli, all the anomalous $\mathrm{U}(1) \mathrm{s}$ have to reside in the visible sector otherwise, as we shall explain later on, the superpotential would not be gauge invariant.

\subsection{Tree-level expressions}

The tree-level Kähler potential takes the form:

$$
K_{\text {tree }}=-\ln \mathcal{V}-\ln (S+\bar{S})-\ln \left(\mathrm{i} \int_{X} \Omega \wedge \bar{\Omega}\right),
$$

where $\mathcal{V}$ is the $\mathrm{CY}$ volume measured in string units, while $\Omega$ is the holomorphic $(3,0)$ form of $X$ that depends implicitly on the $Z$-moduli. The internal volume depends on the $T$-moduli since it looks like:

$$
\mathcal{V}=\frac{1}{6} k_{i j k} t_{i} t_{j} t_{k}=\frac{1}{48} k_{i j k}\left(T_{i}+\bar{T}_{i}\right)\left(T_{j}+\bar{T}_{j}\right)\left(T_{k}+\bar{T}_{k}\right),
$$

where $k_{i j k}=\int_{X} \hat{D}_{i} \wedge \hat{D}_{j} \wedge \hat{D}_{k}$ are the triple intersection numbers of $X$.

The tree-level holomorphic gauge kinetic function for both the visible and hidden sector is given by the dilaton:

$$
f_{\text {tree }}=S \quad \Rightarrow \quad \operatorname{Re}\left(f_{\text {tree }}\right) \equiv g_{4}^{-2}=s .
$$

The tree-level superpotential is generated by the three-form flux $H$ and it reads:

$$
W_{\text {flux }}=\int_{X} H \wedge \Omega
$$

with the correct definition of $H$ including $\alpha^{\prime}$ effects:

$$
H=d B_{2}-\frac{\alpha^{\prime}}{4}[\operatorname{CS}(A)-\operatorname{CS}(\omega)]
$$

where $\operatorname{CS}(A)$ is the Chern-Simons three-form for the gauge connection $A$ :

$$
\mathrm{CS}(A)=\operatorname{Tr}\left(A \wedge d A+\frac{2}{3} A \wedge A \wedge A\right),
$$

and $\operatorname{CS}(\omega)$ is the gravitational equivalent for the spin connection $\omega$.

The VEV of the tree-level superpotential, $W_{0}$, is of crucial importance. Due to the difference with type IIB where one has two three-form fluxes, which can give rise to cancellations among themselves leading to small values of $W_{0}$, in the heterotic case $W_{0}$ is generically of order unity. Hence one experiences two problems:

1. Contrary to type IIB, the heterotic dilaton is not fixed by the flux superpotential, resulting in a supergravity theory which is not of no-scale type. More precisely, the F-term scalar potential:

$$
V_{F}=e^{K}\left(K^{I \bar{J}} D_{I} W D_{\bar{J}} \bar{W}-3|W|^{2}\right),
$$


derived from (2.2) and (2.5) simplifies to:

$$
\begin{aligned}
V_{F} & =e^{K}\left[\sum_{Z} K^{\alpha \bar{\beta}} D_{\alpha} W D_{\bar{\beta}} \bar{W}+\left(K^{S \bar{S}} K_{S} K_{\bar{S}}+\sum_{T} K^{i \bar{j}} K_{i} K_{\bar{j}}-3\right)|W|^{2}\right] \\
& =e^{K}\left(\sum_{Z} K^{\alpha \bar{\beta}} D_{\alpha} W D_{\bar{\beta}} \bar{W}+|W|^{2}\right)
\end{aligned}
$$

since $K^{S \bar{S}} K_{S} K_{\bar{S}}=1$ and $\sum_{T} K^{i \bar{j}} K_{i} K_{\bar{j}}-3=0$. Setting $D_{\alpha} W=0 \forall \alpha=$ $1, \ldots, h^{1,2}(X)$, the scalar potential $(2.9)$ reduces to:

$$
V_{F}=e^{K}\left|W_{0}\right|^{2}=\frac{\left|W_{0}\right|^{2}}{2 s \mathcal{V}},
$$

yielding a run-away for both $s$ and $\mathcal{V}$ if $\left|W_{0}\right| \neq 0$. Given that generically $\left|W_{0}\right| \simeq$ $\mathcal{O}(1)$, it is very hard to balance this tree-level run-away against $S$-dependent nonperturbative effects which are exponentially suppressed in $S$. One could try to do it by considering small values of $s=g_{4 D}^{-2}$ but this would involve a strong coupling limit where control over moduli stabilisation is lost. A possible way to lower $\left|W_{0}\right|$ was proposed in [17] where the authors derived the topological conditions to have fractional Chern-Simons invariants.

2. If $\left|W_{0}\right| \neq 0$, even if it is fractional, one cannot obtain low-energy supersymmetry. In fact, the gravitino mass is given by $m_{3 / 2}=e^{K / 2}\left|W_{0}\right| M_{P}$, and so the invariant quantity $e^{K / 2}\left|W_{0}\right|=\left|W_{0}\right| /(\sqrt{2 s \mathcal{V}})$ has to be of order $10^{-15}$ to have TeV-scale supersymmetry. As we have seen, the $4 \mathrm{D}$ gauge coupling is given by $\alpha_{G U T}^{-1}=g_{s}^{-2} \mathcal{V}$, and so a huge value of the internal volume would lead to a hyper-weak GUT coupling. Note that a very large value of $\mathcal{V}$ cannot be compensated by a very small value of $g_{s}^{-2}$ since we do not want to violate string perturbation theory.

Let us briefly mention that in some particular cases one could have an accidental cancellation among the flux quanta which yields a small $\left|W_{0}\right|$ as suggested in [18]. We stress that in the heterotic case, contrary to type IIB, this cancellation is highly nongeneric, and so it is not very appealing to rely on it to lower $\left|W_{0}\right|$. Hence it would seem that the most promising way to get low-energy supersymmetry is to consider the case where $\left|W_{0}\right|=0$ and generate an exponentially small superpotential only at sub-leading non-perturbative level. This case was considered in [34], where the authors argued that, at tree-level, one can in principle obtain a Minkowski supersymmetric vacuum with all complex structure moduli stabilised and $2\left(h^{1,1}+1\right)$ flat directions corresponding to the dilaton and the Kähler moduli. As explained in section 1, this corresponds to the best case scenario where the gauge bundle is holomorphic only at a non-singular point-like sub-locus in complex structure moduli space.

If instead one focuses on the more general case where $h_{\text {hol }}^{1,2}>0 Z$-moduli are left flat after imposing the requirement of a holomorphic gauge bundle, as we shall show in section 3 , the conditions $D_{Z^{\alpha}} W_{\text {flux }}=0 \forall \alpha=1, \ldots, h_{\text {hol }}^{1,2}$ and $\left|W_{0}\right|=0$ imply that no quantised $H$ flux is turned on, resulting in the impossibility to stabilise the remaining $Z$-moduli. This result 
implies that it is impossible to stabilise the remaining complex structure moduli and the dilaton in two steps with a $Z$-moduli stabilisation at tree-level and a dilaton stabilisation at sub-leading non-perturbative level. In this case there are two possible way-outs:

1. Focus on the case $D_{Z^{\alpha}} W=0 \forall \alpha=1, \ldots, h_{\text {hol }}^{1,2}$ and $\left|W_{0}\right| \neq 0$ so that $H$ can be nontrivial. In this case one has however a dilaton run-away, implying that no moduli can be fixed at tree-level. One needs therefore to add $S$-dependent non-perturbative effects which have to be balanced against the tree-level superpotential to lift the runaway. A small $\left|W_{0}\right|$ could be obtained either considering fractional Chern-Simons invariants or advocating accidental cancellations among the flux quanta.

2. Focus on the case with trivial $H$ so that no scalar potential is generated at treelevel. The dilaton and the complex structure moduli could then be fixed at nonperturbative level via a race-track superpotential generating an exponentially small $W$ which could lead to low-energy supersymmetry. Note that even though $d H=$ $R \wedge R-F \wedge F \neq 0$ for $(0,2)$-models, it is still possible to have $\left|W_{0}\right|=0$ since only the harmonic part of the $H$-flux contributes to this superpotential (see discussion in section 3.1). Hence, moduli stabilisation would have to proceed via a racetrack mechanism involving at least two condensing gauge groups with all moduli appearing in the gauge kinetic functions and/or the prefactors of the non-perturbative terms. Since this is generically not the case for heterotic compactifications, this avenue will not lead to supersymmetric moduli stabilisation except perhaps for a few specific cases. Note that in this case to get a Minkowski supersymmetric vacuum one would have to fine-tune the prefactors of the two (or more) condensates so that $W=0$ at the minimum. Then one would have (under the conditions mentioned above) a set of holomorphic equations for the $Z$-moduli which will always have a solution. However once supersymmetry is broken this option is no-longer available since now one needs to have $W \neq 0$ at the minimum if one is to have any hope of fine-tuning the cosmological constant to zero. However now the equations for the $Z$-moduli are a set of real non-linear equations which are not guaranteed to have a solution.

\subsection{Corrections beyond leading order}

As explained in the previous section, in smooth heterotic compactifications with $h_{\text {hol }}^{1,2}>0$ complex structure moduli not fixed by the holomorphicity of the gauge bundle, these $Z$ moduli cannot be frozen at tree-level by turning on a quantised background flux since this stabilisation would need $\left|W_{0}\right| \neq 0$ which, in turn, would induce a dilaton and volume runaway. Thus, one has to look at any possible correction beyond the leading order expressions. Before presenting a brief summary of the various effects to be taken into account (perturbative and non-perturbative in both $\alpha^{\prime}$ and $g_{s}$ ), let us mention two well-known control issues in heterotic constructions:

- Tension between weak coupling and large volume: In order to have full control over the effective field theory, one would like to stabilise the moduli in a region of field space where both perturbative and higher derivative corrections are small, i.e. respectively 
for $g_{s} \ll 1$ and $\mathcal{V} \gg 1$. However, as we have already pointed out, this can be the case only if the $4 \mathrm{D}$ coupling is hyper-weak, in contrast with phenomenological observations. In fact, we have:

$$
\frac{g_{s}^{2}}{\mathcal{V}}=\alpha_{G U T} \simeq \frac{1}{25}
$$

and so if we require $g_{s} \lesssim 1$, the CY volume cannot be very large, $\mathcal{V} \lesssim 25$, implying that one has never a solid parametric control over the approximations used to fix the moduli.

- Tension between GUT scale and large volume: In heterotic constructions, the unification scale is identified with the Kaluza-Klein scale, $M_{G U T}=M_{K K}$, which cannot be lowered that much below the string scale for $\mathcal{V} \lesssim 25$, resulting in a GUT scale which is generically higher than the value inferred from the 1-loop running of the MSSM gauge couplings. In more detail, the string scale $M_{s} \equiv \ell_{s}^{-1}$ can be expressed in terms of the $4 \mathrm{D}$ Planck scale from dimensional reduction as (see appendix A for an explicit derivation):

$$
M_{s}^{2}=\frac{M_{P}^{2}}{4 \pi \alpha_{G U T}^{-1}} \simeq \frac{M_{P}^{2}}{100 \pi} \simeq\left(1.35 \cdot 10^{17} \mathrm{GeV}\right)^{2} .
$$

In the case of an isotropic compactification, the Kaluza-Klein scale takes the form:

$$
M_{G U T}=M_{K K} \simeq \frac{M_{s}}{\mathcal{V}^{1 / 6}} \gtrsim 8 \cdot 10^{16} \mathrm{GeV} \quad \text { for } \quad \mathcal{V} \lesssim 25,
$$

which is clearly above the phenomenological value $M_{G U T} \simeq 2.1 \cdot 10^{16} \mathrm{GeV}$. On the other hand, anisotropic compactifications with $d$ large dimensions of size $L=x \ell_{s}$ with $x \gg 1$ and $(6-d)$ small dimensions of string size $l=\ell_{s}$, can lower the KaluzaKlein scale:

$$
\operatorname{Vol}(X)=L^{d} l^{6-d}=x^{d} \ell_{s}^{6}=\mathcal{V} \ell_{s}^{6} \Rightarrow M_{G U T}=M_{K K} \simeq \frac{M_{s}}{x} \simeq \frac{M_{s}}{\mathcal{V}^{1 / d}} .
$$

For the case $d=2$, one would get the encouraging result $M_{G U T}=\frac{M_{s}}{\sqrt{\mathcal{V}}} \gtrsim 2.7 \cdot 10^{16} \mathrm{GeV}$.

\subsubsection{Higher derivative effects}

Let us start considering higher derivative effects, i.e. perturbative $\alpha^{\prime}$ corrections to the Kähler potential. In the case of the standard embedding corresponding to $(2,2)$ worldsheet theories, the leading $\alpha^{\prime}$ correction arises at $\mathcal{O}\left(\alpha^{\prime 3}\right) \mathcal{R}^{4}[24]$ and depends on the CY Euler number $\chi(X)=2\left(h^{1,1}-h^{1,2}\right)$. Its form can be derived by substituting the $\alpha^{\prime}$ corrected volume $\mathcal{V} \rightarrow \mathcal{V}+\xi / 2$ into the tree-level expression $(2.2)$ with $\xi=-\zeta(3) \chi(X) /\left(2(2 \pi)^{3}\right)$. Given that $\zeta(3) \simeq 1.2, \xi$ is of the order $\xi \simeq\left(h^{1,2}-h^{1,1}\right) / 200 \simeq \mathcal{O}(1)$ for ordinary CY three-folds with $\left(h^{1,2}-h^{1,1}\right) \simeq \mathcal{O}(100)$. Hence for $\mathcal{V} \simeq \mathcal{O}(20)$, the ratio $\xi /(2 \mathcal{V}) \simeq \mathcal{O}(1 / 40)$ is a small number which justifies the expansion:

$$
K \simeq-\ln \mathcal{V}-\frac{\xi}{2 \mathcal{V}} \quad \Rightarrow \quad K_{\alpha^{\prime 3}}=-\frac{\xi}{2 \mathcal{V}}
$$


As pointed out in [23] however, this is the leading order higher derivative effect only for the standard embedding since $(0,2)$ worldsheet theories admit $\alpha^{\prime}$ corrections already at $\mathcal{O}\left(\alpha^{\prime 2}\right)$ which deform the Kähler form $J$ as:

$$
J \rightarrow J^{\prime}=J+\mathcal{O}\left(\alpha^{\prime}\right) \tilde{h}+\mathcal{O}\left(\alpha^{\prime 2}\right) \tilde{h}^{(2)}+\ldots,
$$

where both $\tilde{h}$ and $\tilde{h}^{(2)}$ are moduli-dependent $(1,1)$-forms which are orthogonal to $J$, i.e. $\int_{X} * J \wedge \tilde{h}=\int_{X} * J \wedge \tilde{h}^{(2)}=0$. Plugging $J^{\prime}$ into the tree-level expression for $K(2.2)$ and then expanding, one finds that the $\mathcal{O}\left(\alpha^{\prime}\right)$ correction vanishes because of the orthogonality between $\tilde{h}$ and $J$ whereas at $\mathcal{O}\left(\alpha^{\prime 2}\right)$ one finds: ${ }^{5}$

$$
K_{\alpha^{\prime 2}}=\frac{1}{2 \mathcal{V}} \int_{X} * \tilde{h} \wedge \tilde{h}=\frac{\|\tilde{h}\|^{2}}{2 \mathcal{V}}
$$

Note that the correction (2.17) is generically leading with respect to (2.15) since (2.17) should be more correctly rewritten as:

$$
K_{\alpha^{\prime 2}}=\frac{g}{\mathcal{V}^{2 / 3}} \quad \text { with } \quad g \equiv \frac{\|\tilde{h}\|^{2}}{2 \mathcal{V}^{1 / 3}}=-\frac{1}{2 \mathcal{V}^{1 / 3}} \int_{X} J \wedge \tilde{h} \wedge \tilde{h} \geq 0,
$$

where $g$ is a homogeneous function of the Kähler moduli of degree 0 given that $J$ scales as $J \sim \mathcal{V}^{1 / 3}$ and $\tilde{h}$ does not depend on $\mathcal{V}$. As an illustrative example, let us consider the simplest Swiss-cheese CY $X$ with one large two-cycle $t_{b}$ and one small blow-up mode $t_{s}$ so that $J=t_{b} \hat{D}_{b}-t_{s} \hat{D}_{s}$ and the volume reads:

$$
\mathcal{V}=k_{b} t_{b}^{3}-k_{s} t_{s}^{3}>0 \quad \text { for } \quad 0 \leq \frac{t_{s}}{t_{b}}<\left(\frac{k_{b}}{k_{s}}\right)^{1 / 3}
$$

In the limit $k_{b} t_{b}^{3} \gg k_{s} t_{s}^{3}$, the function $g$ then becomes (considering, without loss of generality, $\tilde{h}$ as moduli-independent):

$g=c_{b}+c_{s} \frac{t_{s}}{t_{b}} \geq 0 \quad$ with $\quad c_{b}=-\frac{1}{2 k_{b}^{1 / 3}} \int_{X} \hat{D}_{b} \wedge \tilde{h} \wedge \tilde{h} \quad$ and $\quad c_{s}=\frac{1}{2 k_{b}^{1 / 3}} \int_{X} \hat{D}_{s} \wedge \tilde{h} \wedge \tilde{h}$.

The sign of $c_{b}$ and $c_{s}$ can be constrained as follows. In the limit $t_{s} / t_{b} \rightarrow 0, g$ reduces to $g=c_{b}=\left|c_{b}\right| \geq 0$. On the other hand, requiring that $g$ is semi-positive definite for any point in Kähler moduli space one finds:

$$
c_{s}=-\left|c_{b}\right|\left(\frac{k_{s}}{k_{b}}\right)^{1 / 3}+|\kappa|,
$$

where $|\kappa|$ is a semi-positive definite quantity.

\footnotetext{
${ }^{5}$ In looking at the derivation of the correction at $\mathcal{O}\left(\alpha^{\prime 2}\right)$ in [23], one may wonder about the rôle of field redefinitions. The fact that the corrected Kähler potential $K^{\prime}$ can be written in terms of $J^{\prime}$ as a function of $\int J^{\prime} \wedge J^{\prime} \wedge J^{\prime}$ alone, just the same way as the tree-level $K$ in terms of $J$, may imply that a field redefinition of the Kähler form may actually fully absorb the correction at $\mathcal{O}\left(\alpha^{\prime 2}\right)$. To this end, the observation in [23] that the generically non-vanishing string 1-loop corrections in type IIB appearing at $\mathcal{O}\left(\alpha^{\prime 2}\right)$ are S-dual to the heterotic correction, provides additional evidence for the existence of this term.
} 


\subsubsection{Loop effects}

Let us now focus on $g_{s}$ perturbative effects which can modify both the Kähler potential and the gauge kinetic function. The exact expression of the string loop corrections to the Kähler potential is not known due to the difficulty in computing string scattering amplitudes on CY backgrounds. However, in the case of type IIB compactifications, these corrections have been argued to be sub-leading compared to $\alpha^{\prime}$ effects by considering the results for simple toroidal orientifolds [48] and trying to generalise them to arbitrary CY backgrounds [49, 50]. Following [50], we shall try to estimate the behaviour of string loop corrections to the scalar potential by demanding that these match the Coleman-Weinberg potential:

$$
V_{g_{s}} \simeq \Lambda^{2} \operatorname{Str} M^{2} \simeq m_{3 / 2}^{2} M_{K K}^{2} \simeq \frac{|W|^{2}}{2 s} \frac{M_{P}^{4}}{\mathcal{V}^{2(1+1 / d)}}
$$

where we took the cut-off scale $\Lambda=M_{K K}$ and we considered $d$ arbitrary large dimensions. Note that these effects are indeed subdominant with respect to the $\alpha^{\prime}$ ones for large volume since the $\mathcal{O}\left(\alpha^{\prime 2}\right)$ and $\mathcal{O}\left(\alpha^{\prime 3}\right)$ corrections, (2.18) and (2.15), give respectively a contribution to the scalar potential of the order $V_{\alpha^{\prime 2}} \simeq|W|^{2} / \mathcal{V}^{5 / 3}$ and $V_{\alpha^{\prime 3}} \simeq|W|^{2} / \mathcal{V}^{2}$, whereas the $g_{s}$ potential (2.22) scales as $V_{g_{s}} \simeq|W|^{2} / \mathcal{V}^{7 / 3}$ for the isotropic case with $d=6$ and $V_{g_{s}} \simeq|W|^{2} / \mathcal{V}^{3}$ for the anisotropic case with $d=2$. Due to this subdominant behaviour of the string loop effects, we shall neglect them in what follows.

String loops correct also the gauge kinetic function (2.4). The 1-loop correction has a different expression for the visible and hidden $E_{8}$ sectors [22]:

$$
f_{\text {vis }}=S+\frac{\beta_{i}}{2} T_{i}, \quad f_{\text {hid }}=S-\frac{\beta_{i}}{2} T_{i},
$$

where:

$$
\beta_{i}=\frac{1}{4 \pi} \int_{X}\left(c_{2}\left(V_{\text {vis }}\right)-c_{2}\left(V_{\text {hid }}\right)\right) \wedge \hat{D}_{i} .
$$

\subsubsection{Non-perturbative effects}

The $4 \mathrm{D}$ effective action receives also non-perturbative corrections in both $\alpha^{\prime}$ and $g_{s}$. The $\alpha^{\prime}$ effects are worldsheet instantons wrapping an internal two-cycle $T_{i}$. These give a contribution to the superpotential of the form:

$$
W_{\mathrm{wi}}=\sum_{j} B_{j} e^{-b_{i j} T_{i}} .
$$

Note that these contributions arise only for $(0,2)$ worldsheet theories whereas they are absent in the case of the standard embedding. On the other hand, $g_{s}$ non-perturbative effects include gaugino condensation and NS5 instantons. In the case of gaugino condensation in the hidden sector group, the resulting superpotential looks like:

$$
W_{\mathrm{gc}}=\sum_{j} A_{j} e^{-a_{j} f_{\mathrm{hid}}}=\sum_{j} A_{j} e^{-a_{j}\left(S-\frac{\beta_{i}}{2} T_{i}\right)},
$$

where in the absence of hidden sector $\mathrm{U}(1)$ factors, all the hidden sector gauge groups have the same gauge kinetic function. Finally, NS5 instantons wrapping the whole CY manifold would give a sub-leading non-perturbative superpotential suppressed by $e^{-\mathcal{V}} \ll 1$, and so we shall neglect them. 


\subsection{Moduli-dependent Fayet-Iliopoulos terms}

As already pointed out, we shall allow line bundles in the visible sector where we turn on a vector bundle of the form $V_{\text {vis }}=U_{\text {vis }} \bigoplus_{\kappa} \mathcal{L}_{\kappa}$. The presence of anomalous $\mathrm{U}(1)$ factors induces $\mathrm{U}(1)$ charges for the moduli in order to cancel the anomalies and gives rise to moduli-dependent Fayet-Iliopoulos (FI) terms. In particular, the charges of the Kähler moduli and the dilaton under the $\kappa$-th anomalous $\mathrm{U}(1)$ read:

$$
q_{T_{i}}^{(\kappa)}=4 c_{1}^{i}\left(\mathcal{L}_{\kappa}\right) \quad \text { and } \quad q_{s}^{(\kappa)}=2 \gamma_{(\kappa)}=2 \beta_{i} c_{1}^{i}\left(\mathcal{L}_{\kappa}\right),
$$

so that the FI-terms become [22]:

$$
\xi_{(\kappa)}=-q_{T_{i}}^{(\kappa)} \frac{\partial K}{\partial T_{i}}-q_{s}^{(\kappa)} \frac{\partial K}{\partial S}=\frac{c_{1}^{i}\left(\mathcal{L}_{\kappa}\right)}{\mathcal{V}} k_{i j k} t_{j} t_{k}+\frac{\gamma_{(\kappa)}}{s} .
$$

Note that the dilaton-dependent term in the previous expression is a 1-loop correction to the FI-terms which at tree-level depend just on the Kähler moduli. The final D-term potential takes the form:

$$
V_{D}=\sum_{\kappa} \frac{\xi_{(\kappa)}^{2}}{\operatorname{Re}\left(f_{(\kappa)}\right)} .
$$

From the expressions (2.27) for the U(1)-charges of the moduli, we can now check the $\mathrm{U}(1)$-invariance of the non-perturbative superpotentials (2.25) and (2.26). In the absence of charged matter fields, the only way to obtain a gauge invariant worldsheet instanton is to choose the gauge bundle such that all the $T_{i}$ appearing in $W_{\mathrm{wi}}$ are not charged, i.e. $c_{1}^{i}\left(\mathcal{L}_{\kappa}\right)=0 \forall \kappa$ and $\forall i$. The superpotential generated by gaugino condensation is instead automatically $\mathrm{U}(1)$-invariant by construction since all the anomalous $\mathrm{U}(1) \mathrm{s}$ are in the visible sector whereas gaugino condensation takes place in the hidden sector. Thus, the hidden sector gauge kinetic function is not charged under any anomalous $\mathrm{U}(1)$ :

$$
q_{f_{\text {hid }}}^{(\kappa)}=q_{s}^{(\kappa)}-\frac{\beta_{i}}{2} q_{T_{i}}^{(\kappa)}=2\left(\gamma_{(\kappa)}-\beta_{i} c_{1}^{i}\left(\mathcal{L}_{\kappa}\right)\right)=0 .
$$

Before concluding this section, we recall that in supergravity the D-terms are proportional to the $\mathrm{F}$-terms for $W \neq 0$. In fact, the total $\mathrm{U}(1)$-charge of the superpotential $W$ is given by $q_{W}^{(\kappa)}=q_{i}^{(\kappa)} W_{i} / W=0$, and so one can write:

$$
\xi_{(\kappa)}=-q_{i}^{(\kappa)} K_{i}=-q_{i}^{(\kappa)} \frac{D_{i} W}{W}=-q_{i}^{(\kappa)} \frac{e^{-K / 2}}{W} K_{i j} \bar{F}^{\bar{j}},
$$

where the F-terms are defined as $F^{i}=e^{K / 2} K^{i \bar{j}} D_{\bar{j}} \bar{W}$. Therefore if all the F-terms are vanishing with $W \neq 0$, the FI-terms are also all automatically zero without giving rise to independent moduli-fixing relations.

\section{Supersymmetric vacua}

In this section, we shall perform a systematic discussion of heterotic supersymmetric vacua starting from an analysis of the tree-level scalar potential and then including corrections beyond the leading order expressions. 


\subsection{Tree-level scalar potential}

In [20], Strominger analysed the 10D equations of motion and worked out the necessary and sufficient conditions to obtain $\mathcal{N}=1$ supersymmetry in $4 \mathrm{D}$ assuming a $10 \mathrm{D}$ space-time of the form $M \times X$ where $M$ is a maximally symmetric $4 \mathrm{D}$ space-time and $X$ is a compact $6 \mathrm{D}$ manifold:

1. $M$ is Minkowski;

2. $X$ is a complex manifold, i.e. the Nijenhuis tensor has to vanish;

3. There exists one globally defined holomorphic $(3,0)$-form $\Omega$ which is closed, i.e. $d \Omega=$ 0 , and whose norm is related to the complex structure $(1,1)$-form $J$ as (up to a constant): ${ }^{6}$

$$
d^{\dagger} J=i(\partial-\bar{\partial}) \ln \|\Omega\|
$$

4. The background gauge field $F$ has to satisfy the Hermitian Yang-Mills equations:

$$
F_{(0,2)}=F_{(2,0)}=0 \quad \text { and } \quad g^{i \bar{j}} F_{i \bar{j}}=0
$$

5. The dilaton $\phi$ and the warp factor $A$ have to satisfy (again up to a constant): ${ }^{7}$

$$
\phi(y)=A(y)=\frac{1}{8} \ln \|\Omega\|(y)
$$

6. The background three-form flux is given by:

$$
H=-\frac{i}{2}(\partial-\bar{\partial}) J
$$

together with the Bianchi identity:

$$
d H=-\frac{\alpha^{\prime}}{4}[\operatorname{tr}(F \wedge F)-\operatorname{tr}(R \wedge R)]
$$

Some of the conditions listed above can be reformulated also in terms of constraints on the five torsional classes $\mathcal{W}_{i}, i=1, \ldots, 5$ (for a review see $[1,40]$ ). The second condition corresponds to $\mathcal{W}_{1}=\mathcal{W}_{2}=0$ implying that the torsional class $\tau$ belongs to the space $\tau \in \mathcal{W}_{3} \oplus \mathcal{W}_{4} \oplus \mathcal{W}_{5}$. This is the case of 'Hermitian manifolds'. Moreover, the third condition above gives $\mathcal{W}_{5}=-2 \mathcal{W}_{4}=d \ln \|\Omega\|$ implying that both $\mathcal{W}_{4}$ and $\mathcal{W}_{5}$ are exact real 1 -forms. We shall focus on the simplest solution to $2 \mathcal{W}_{4}+\mathcal{W}_{5}=0$ which is $\mathcal{W}_{4}=\mathcal{W}_{5}=0$ corresponding to the case of 'special-Hermitian manifolds' where the dilaton and the warp factor are constant [40]. More general solutions involve a non-constant dilaton profile in the extra dimensions and $\mathcal{W}_{i} \neq 0$ for $i=4,5$ but we shall not consider this option [40].

\footnotetext{
${ }^{6}$ The adjoint operator $d^{\dagger}$ can be defined from the inner product $\langle\omega, \sigma\rangle=\int_{X} \omega \wedge * \sigma$ as $\left\langle\omega_{p}, d \omega_{p-1}\right\rangle=$ $\left\langle d^{\dagger} \omega_{p}, \omega_{p-1}\right\rangle$. For an even dimensional manifold, as we have here, $d^{\dagger}=-* d *$.

${ }^{7}$ We are writing the total metric as $d s^{2}=e^{2 A(y)}\left(g_{\mu \nu}(x) d x^{\mu} d x^{\nu}+g_{i j}(y) d y^{i} d y^{j}\right)$.
} 
Let us comment on the implications of the last Strominger condition (3.4) which for constant dilaton can be rewritten as $H=-\frac{1}{2} * d J$. Using the Hodge decomposition theorem, the three-form $H$ can be expanded uniquely as:

$$
H=H_{\text {harm }}+H_{\text {exact }}+H_{\text {co-exact }},
$$

where $H_{\text {harm }}$ is a harmonic form, $H_{\text {exact }}$ is an exact form and $H_{\text {co-exact }}$ is a co-exact form which are all orthogonal to each other. Given that $* d J=-d^{\dagger} * J,(3.4)$ implies that $H$ is a co-exact form, and so $H_{\text {harm }}=H_{\text {exact }}=0$. Moreover, since $d J$ is a $(2,1)+(1,2)$ form, (3.4) implies that the $(3,0)+(0,3)$ component of $H_{\text {co-exact }}$ is zero while the $(2,1)+$ $(1,2)$ component breaks the Kähler condition $d J=0$. However this happens only at $\mathcal{O}\left(\alpha^{\prime}\right)$. In fact, the general expression of the $H$-flux is:

$$
H=H_{\text {flux }}+d B_{2}-\frac{\alpha^{\prime}}{4}[\operatorname{CS}(A)-\operatorname{CS}(\omega)]
$$

where $H_{\text {flux }}$ is a harmonic piece and the combination of Chern-Simons three-forms can also be decomposed as:

$$
[\mathrm{CS}(A)-\mathrm{CS}(\omega)]=\mathrm{CS}_{\text {harm }}+\mathrm{CS}_{\text {exact }}+\mathrm{CS}_{\text {co-exact }} .
$$

Comparing the two expressions for $H,(3.6)$ and (3.7), we have (due to the uniqueness of the Hodge decomposition):

$$
H_{\text {harm }}=H_{\text {flux }}-\frac{\alpha^{\prime}}{4} \mathrm{CS}_{\text {harm }}, \quad H_{\text {exact }}=d B_{2}-\frac{\alpha^{\prime}}{4} \mathrm{CS}_{\text {exact }}, \quad H_{\text {co-exact }}=-\frac{\alpha^{\prime}}{4} \mathrm{CS}_{\text {co-exact }} .
$$

Then the relation (3.4) takes the form:

$$
\frac{\alpha^{\prime}}{2} \mathrm{CS}_{\mathrm{co}-\mathrm{exact}}=* d J
$$

showing exactly that the Kähler condition $d J=0$ is violated at $O\left(\alpha^{\prime}\right)$. Note that this would be the case for the non-standard embedding where $\mathrm{CS}_{\mathrm{co}-\text { exact }} \neq 0$ contrary to the less generic situation of the standard embedding where the Chern-Simons piece vanishes. Taking the exterior derivative of (3.9) we recover the Bianchi identity (3.5) which now looks like:

$$
d * d J=\frac{\alpha^{\prime}}{2}[\operatorname{tr}(F \wedge F)-\operatorname{tr}(R \wedge R)]
$$

This 10D analysis can also be recast in terms of an effective potential which can be written as a sum of BPS-like terms and whose minimisation reproduces the conditions above [36, 42-44]. Furthermore, some of these conditions can be re-derived as F- or Dterm equations of $4 \mathrm{D}$ supergravity, which could lead to the stabilisation of some of the moduli in a Minkowski vacuum. For example, it has been shown in [36, 44], that the second equation in (3.2) is equivalent to a D-term condition since:

$$
*_{6} 1 \cdot g^{i \bar{j}} F_{i \bar{j}}=\frac{1}{2} F \wedge J \wedge J
$$


This D-term condition holds for general non-Abelian gauge fields. If we restrict to Abelian fluxes and integrate the above condition over the CY, this reproduces the tree-level expression for the Fayet-Iliopoulos terms given in (2.28). If we expand the Abelian fluxes as $F_{(\kappa)}=c_{1}^{i}\left(\mathcal{L}_{\kappa}\right) \hat{D}_{i}$ together with $J=t^{j} \hat{D}_{j}$ we obtain:

$$
\xi_{(\kappa)}=\frac{1}{\mathcal{V}} \int_{X} F_{(\kappa)} \wedge J \wedge J=\frac{c_{1}^{i}\left(\mathcal{L}_{\kappa}\right)}{\mathcal{V}} k_{i j k} t_{j} t_{k}
$$

which reproduces exactly the tree-level part of (2.28).

Regarding the F-terms, as we have seen in section 2.1, the starting point is the expression of the flux superpotential which has been inferred in [45] by comparing the dimensional reduction of the $10 \mathrm{D}$ coupling of $H$ to the gravitino mass term in the $4 \mathrm{D}$ supergravity action. The final result is: ${ }^{8}$

$$
W_{\text {flux }}=\int_{X} H \wedge \Omega=\int_{X} H_{\text {harm }} \wedge \Omega .
$$

Note that only the harmonic component of $H$ contributes to $W_{\text {flux }}$. The harmonic piece $H_{\text {harm }}$ can be expanded in a basis of harmonic $(3,0)$ - and $(1,2)$-forms as:

$$
H_{\text {harm }}=\bar{a}(\bar{Z}) \Omega(Z)+b^{\alpha}(Z, \bar{Z}) \chi_{\alpha}(Z, \bar{Z})+\text { c.c. }, \quad \alpha=1, \ldots, h^{1,2}(X) .
$$

The same $H_{\text {harm }}$, together with the holomorphic $(3,0)$-form $\Omega$, can also be expanded in a symplectic basis of harmonic three-forms $\left(\alpha_{p}, \beta^{q}\right)$ such that $\int_{X} \alpha_{p} \wedge \alpha_{q}=\int_{X} \beta^{p} \wedge \beta^{q}=0$ and $\int_{X} \alpha_{p} \wedge \beta^{q}=\delta_{p}^{q}$ with $p, q=0, \ldots, h^{1,2}(X)$ :

$$
H_{\text {harm }}=e^{p} \alpha_{p}-m_{q} \beta^{q} \quad \text { and } \quad \Omega(Z)=Z^{p} \alpha_{p}-G_{q}(Z) \beta^{q},
$$

where $G_{q}(Z)=\partial_{Z^{q}} G(Z)$ with $G(Z)$ a homogeneous function of degree 2. Note that $\alpha_{p}$ and $\beta^{q}$ do not depend on the complex structure moduli $Z^{\alpha}$ which are defined by the expansion of $\Omega$ in (3.15). If $\left(A_{p}, B^{q}\right)$ is the dual symplectic basis of 3-cycles such that $A_{p} \cap A_{q}=B^{p} \cap B^{q}=0$ and $A_{p} \cap B^{q}=\delta_{p}^{q}$, we have (choosing units such that $2 \pi \sqrt{\alpha^{\prime}}=1$ ):

$$
\int_{B^{p}} H_{\mathrm{harm}}=\int_{X} H_{\mathrm{harm}} \wedge \beta^{p}=\int_{X}\left(e^{r} \alpha_{r}-m_{q} \beta^{q}\right) \wedge \beta^{p}=e^{p},
$$

and similarly $m_{q}=\int_{A_{q}} H_{\text {harm. }}$. The quantities $e^{p}$ and $m_{q}$ are integer flux quanta.

The expansion of the flux superpotential (3.13) is then given by:

$$
\begin{aligned}
W_{\text {flux }}(Z) & =\int_{X} H_{\text {harm }} \wedge \Omega=a(Z) \int_{X} \bar{\Omega}(\bar{Z}) \wedge \Omega(Z) \\
& =\mathrm{i} a(Z)=m_{q} Z^{q}-e^{p} G_{p}(Z),
\end{aligned}
$$

\footnotetext{
${ }^{8}$ In [42] and [44] it is suggested that the complete expression for $W$ should more appropriately be $W=\int_{X}\left(H+\frac{i}{2} d J\right) \wedge \Omega$, similarly to the type IIB case where one has the RR flux in addition to the $H$-flux. Integrating by parts, the new piece can be rewritten as $\int_{X} J \wedge d \Omega$ which clearly vanishes since $d \Omega=0$. However, if one considers the case where $d \Omega \neq 0$, i.e. where supersymmetry is broken directly at the $10 \mathrm{D}$ level, this integral would still be zero if the internal manifold is complex since $d J$ is of Hodge type $(2,1)+(1,2)$ while $\Omega$ is $(3,0)$. Thus this term can play a useful rôle only for non-complex manifolds with broken supersymmetry. Due to the difficulty to study this case in a controlled way, we shall not consider it and neglect this additional piece.
} 
where we normalised $\int_{X} \Omega \wedge \bar{\Omega}=-\mathrm{i}$ and used the fact that $* \Omega=-\mathrm{i} \Omega$ and the orthogonality of the different Hodge components of $H$.

Let us now evaluate the complex structure F-terms $D_{Z^{\alpha}} W_{\text {flux }}=\partial_{Z^{\alpha}} W_{\text {flux }}+W_{\text {flux }} \partial_{Z^{\alpha}} K$. Using the fact that (see for example [51]):

$$
\partial_{Z^{\alpha}} \Omega=k_{\alpha}(Z, \bar{Z}) \Omega+\chi_{\alpha}, \quad \partial_{Z^{\alpha}} K=-k_{\alpha}(Z, \bar{Z}),
$$

and:

$$
K_{\alpha \bar{\beta}} \equiv \partial_{Z^{\alpha}} \partial_{\bar{Z} \bar{\beta}} K=\frac{\int_{X} \chi_{\alpha} \wedge \bar{\chi}_{\bar{\beta}}}{\int_{X} \Omega \wedge \bar{\Omega}}=\mathrm{i} \int_{X} \chi_{\alpha} \wedge \bar{\chi}_{\bar{\beta}}
$$

and expanding a generic element of the basis of harmonic $(2,1)$-forms as $\chi_{\alpha}(Z, \bar{Z})=$ $f_{\alpha}^{p}(Z, \bar{Z}) \alpha_{p}-g_{q, \alpha}(Z, \bar{Z}) \beta^{q}$, we find:

$$
\begin{aligned}
D_{Z^{\alpha}} W_{\text {flux }} & =\int_{X} H_{\text {harm }} \wedge \chi_{\alpha}=b^{\bar{\beta}}(Z, \bar{Z}) \int_{X} \bar{\chi}_{\bar{\beta}} \wedge \chi_{\alpha} \\
& =\mathrm{i} b_{\alpha}(Z, \bar{Z})=m_{q} f_{\alpha}^{q}(Z, \bar{Z})-e^{p} g_{p, \alpha}(Z, \bar{Z}),
\end{aligned}
$$

where we used again the orthogonality of the different Hodge components of $H$ and the fact that $* \chi_{\alpha}=\mathrm{i} \chi_{\alpha}$. On the other hand, the dilaton and Kähler moduli F-terms look like:

$$
D_{S} W_{\text {flux }}=W_{\text {flux }} \partial_{S} K=-\mathrm{i} \frac{a}{2 s} \quad \text { and } \quad D_{T_{i}} W_{\text {flux }}=W_{\text {flux }} \partial_{T_{i}} K=-\mathrm{i} \frac{a}{4 \mathcal{V}} k_{i j k} t_{j} t_{k} .
$$

Due to the no-scale cancellation, these F-terms give rise to a scalar potential which is positive definite and reads:

$$
V=e^{K}\left(\sum_{Z} K^{\alpha \bar{\beta}} D_{\alpha} W D_{\bar{\beta}} \bar{W}+|W|^{2}\right)=\frac{1}{2 s \mathcal{V}}\left(\sum_{Z} K^{\alpha \bar{\beta}} b_{\alpha} \bar{b}_{\bar{\beta}}+|a|^{2}\right) .
$$

Let us now set all the F-terms to zero and see what they correspond to:

- $D_{Z^{\alpha}} W_{\text {flux }}=0$ implies that the $(2,1)+(1,2)$ component of $H_{\text {harm }}$ is zero.

- $D_{S} W_{\text {flux }}=0$ and $D_{T_{i}} W_{\text {flux }}=0$ imply that the $(3,0)+(0,3)$ component of $H_{\text {harm }}$ should also be zero, i.e. $W_{0} \equiv\left\langle W_{\text {flux }}\right\rangle=0$, if one wants to avoid solutions with a dilaton run-away $(s \rightarrow \infty)$ or where the internal space decompactifies $(\mathcal{V} \rightarrow \infty)$.

Combining these two solutions, one has that the total harmonic piece of $H$ should vanish and is of course consistent with the Strominger condition (3.4). An important question to ask now is whether these conditions allow for the fixing of some moduli. The answer is no. Let us see why.

The first condition $D_{Z^{\alpha}} W=0$ appears to fix the complex structure moduli supersymmetrically since one obtains as many equations, $b_{\alpha}(Z, \bar{Z})=0$, as the number of unknowns (assuming that the $2 h^{1,2}$ real equations have solutions for some sets of values of the $2 h^{1,2}+2$ fluxes). The second condition $W_{0} \equiv\left\langle W_{\text {flux }}\right\rangle=\mathrm{i} a(Z)=0$ could then be satisfied by an appropriate choice of flux quanta.

However the two conditions $D_{Z^{\alpha}} W=0 \Leftrightarrow b_{\alpha}(Z, \bar{Z})=0 \forall \alpha$ and $W_{0}=0 \Leftrightarrow a(Z)=0$ imply from (3.14) that $H_{\text {harm }}=0$. Given that $H$ does not depend on the complex structure 
moduli, this implies that all flux quanta are zero. In turn, (3.17) and (3.18) are both identically zero, and so no potential for the $Z$-moduli is developed. Therefore no moduli, not even the complex structure ones, can be stabilised at tree-level by using quantised background fluxes. ${ }^{9}$ In particular, this implies that one cannot perform a two-step stabilisation (similarly to type IIB) where at tree-level the $Z$-moduli are fixed supersymmetrically while the $S$ - and $T$-moduli are kept flat by tuning $W_{0}=0$, and then these remaining moduli are lifted by quantum corrections. As we have already pointed out in section 2.1, we shall avoid this problem by considering in the next section situations with non-zero flux quanta which allow to fix the $Z$-moduli with $W_{0} \neq 0$. The dilaton and volume runaway is then prevented by scanning over integral and fractional fluxes which give a value of $W_{0}$ small enough to compete with non-perturbative effects. Hence the system becomes stable only when non-perturbative effects are included, implying that all the moduli get stabilised beyond tree-level.

\subsubsection{Chern-Simons action and gauge bundle moduli}

In this section, we shall show that also the first equation in (3.2), i.e. $F_{(0,2)}=F_{(2,0)}=0$, can be derived from an F-term condition in $4 \mathrm{D}$ supergravity. This requires a brief discussion of gauge bundle moduli. Let us focus on the Chern-Simons piece of the flux superpotential:

$$
W_{\mathrm{CS}}[A]=\int_{X} \operatorname{Tr}\left(A \wedge d A+\frac{2}{3} A \wedge A \wedge A\right) \wedge \Omega .
$$

In the previous expression $A$ is a function of both $x$ and $y$, i.e. non-compact and compact coordinates respectively, but the differentiation is just $d=d y^{m} \partial_{m}$ since we are only interested in the contribution to the $4 \mathrm{D}$ scalar potential. We shall now write the gauge potential as:

$$
A(x, y)=A_{0}(y)+A_{\text {def }}(x, y)
$$

where $A_{0}$ is a background contribution independent of $x$ and $A_{\text {def }}$ is a generic deformation which can be parameterised as:

$$
A_{\mathrm{def}}(x, y)=\sum_{I=1}^{\infty} C_{I}(x) \omega^{I}(y),
$$

where $C_{I}$ are $4 \mathrm{D}$ scalar fields and $\omega^{I}$ are an infinite set of 1-forms living on $X$ and valued in the adjoint representation of the structure group of the gauge bundle defined by $A_{0} \cdot{ }^{10}$

The superpotential (3.21) then becomes the sum of a constant, a linear, a quadratic and a cubic term in the $C$ 's:

$$
W_{\mathrm{CS}}=W_{\mathrm{CS},(0)}+W_{\mathrm{CS},(1)}^{I} C_{I}+W_{\mathrm{CS},(2)}^{I J} C_{I} C_{J}+W_{\mathrm{CS},(3)}^{I J K} C_{I} C_{J} C_{K},
$$

\footnotetext{
${ }^{9}$ The corresponding situation in type IIB is very different since there are two types of fluxes and the effective flux $G_{3}$ is complex [41].

${ }^{10}$ We expect the set of 1 -forms $\omega^{I}$ to be discrete since they will be solutions to an elliptic differential equation on the compact manifold.
} 
with (for notational simplicity we dropped the trace symbol):

$$
\begin{aligned}
& W_{\mathrm{CS},(0)}=W_{\mathrm{CS}}\left[A_{0}\right], \quad W_{\mathrm{CS},(1)}^{I}=2 \int_{X} \omega^{I} \wedge F_{0} \wedge \Omega, \\
& W_{\mathrm{CS},(2)}^{I J}=\int_{X} \omega^{I} \wedge \bar{D}_{0} \omega^{J} \wedge \Omega, \quad W_{\mathrm{CS},(3)}^{I J K}=\frac{2}{3} \int_{X} \omega^{I} \wedge \omega^{J} \wedge \omega^{K} \wedge \Omega,
\end{aligned}
$$

where the gauge covariant derivative $\bar{D}_{0}$ is defined as $\bar{D}_{0} \omega_{(0, p)}=\bar{\partial} \omega_{(0, p)}+A_{0} \wedge \omega_{(0, p)}-$ $(-1)^{p} \omega_{(0, p)} \wedge A_{0}$ for an arbitrary $(0, p)$-form $\omega_{(0, p)}$. Note that in order to derive these expressions we used $d \Omega=0$, the anti-commutativity of $d$ and 1-forms and the cyclicity of the trace. As we have argued earlier, classically the total superpotential $W$ should be zero at the minimum (for all the moduli), and so the F-term equation for the bundle moduli $C_{I}$ is:

$$
0=F_{C_{I}}=\frac{\partial W_{\mathrm{CS}}}{\partial C_{I}}=W_{\mathrm{CS},(1)}^{I}+2 W_{\mathrm{CS},(2)}^{I J} C_{J}+2 W_{\mathrm{CS},(3)}^{I J K} C_{J} C_{K}
$$

If $A_{\text {def }}$ is a small deformation of the background $A_{0}$, i.e. $C_{I}(x)=\varepsilon_{I}(x)$, then these F-term equations can be solved order by order in $\varepsilon$, obtaining:

- At zeroth order $F_{C_{I}}=0$ gives $W_{\mathrm{CS},(1)}^{I}=0 \forall I$ which from (3.25) implies that the (0,2)component of the unperturbed field strength $F_{0}$ has to vanish. Hence we recover the holomorphic Yang-Mills equation $F_{0,(0,2)}=0$ which determines (given a complex structure) $A_{0}$ to be a flat $(0,1)$ connection. This bundle, which we call $Q_{0}$, then determines the exterior derivative operator $\bar{D}_{0}$.

- At linear order $F_{C_{I}}=0$ implies (see the expression of $W_{\mathrm{CS},(2)}^{I J}$ in (3.26)):

$$
W_{\mathrm{CS},(2)}^{I J} C_{J}=0 \quad \forall I \quad \Leftrightarrow \quad C_{J} \bar{D}_{0} \omega^{J}=0 .
$$

This equation has two possible solutions:

1. $\bar{D}_{0} \omega^{i}=0 \quad \forall C_{i} \quad$ for $i=1, \ldots, N$

2. $\bar{D}_{0} \omega^{\iota} \neq 0 \quad C_{\iota}=0 \quad$ for $\iota=N+1, \ldots, \infty$

The first solution defines the gauge bundle moduli which parameterise all possible deformations of the background that keep the gauge bundle holomorphic. These first order deformations correspond to $\omega^{i} \in H^{1}\left(\operatorname{End}\left(Q_{0}\right)\right)$ where $N \equiv \operatorname{dim}\left(H^{1}\left(\operatorname{End}\left(Q_{0}\right)\right)\right)$ which is expected to be finite though it may change as one varies the complex structure since the equations determining the $(0,1)$-forms $\omega^{i}$ depend on the $Z$-moduli. Hence $N$ is a function of the $Z$-moduli, i.e. $N=N(Z)$. If $N=0$ for $Z=Z_{0}$, then if the complex structure moduli can be stabilised via the fluxes exactly at $Z=Z_{0}$, the absence of any gauge bundle moduli is guaranteed (see $[37,38]$ for similar considerations). Conversely, the equation $\bar{D}_{0} \omega^{i}(Z)=0$ could be used as a mechanism to reduce the number of complex structure moduli, or even to fix all of them, if the $C_{i}$ 's develop non-zero VEVs due to D-terms or higher order terms in $W$ [33-35]. We denoted as $h_{\text {hol }}^{1,2}$ the number of $Z$-moduli unconstrained by the equation $\bar{D}_{0} \omega^{i}(Z)=0$, which represents the dimensionality of the sub-locus in complex structure moduli space 


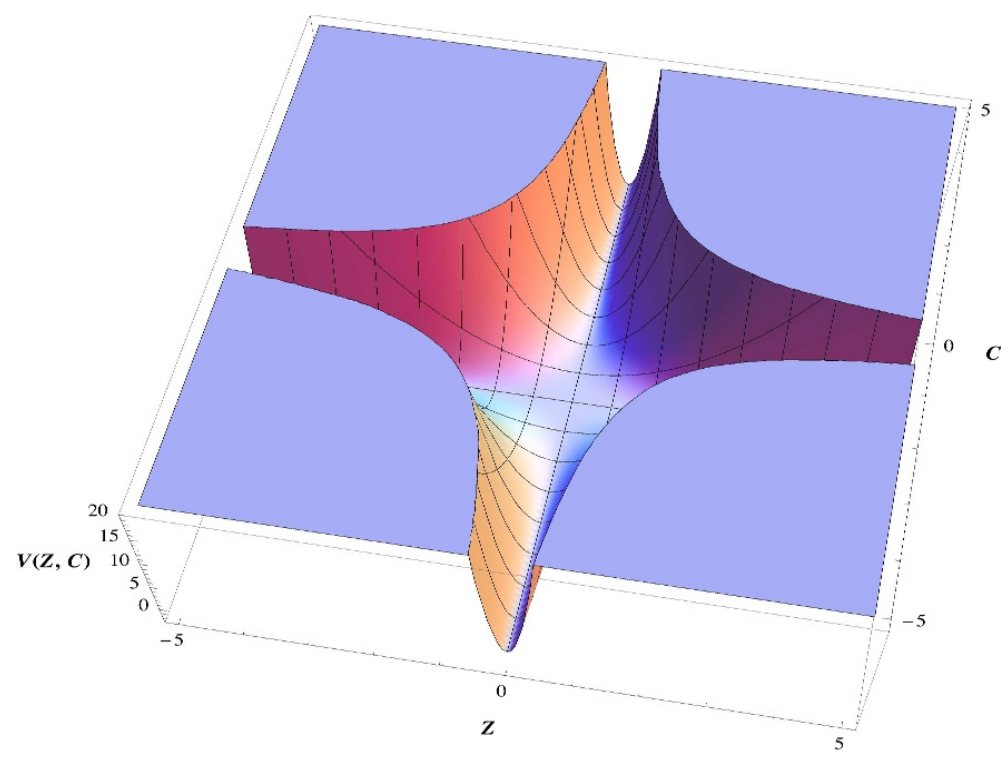

Figure 1. Sketch of the leading order scalar potential $V \sim\left|\partial_{C} W\right|^{2}+\ldots=|Z|^{2}|C|^{2}+\ldots$ as a function of the complex structure moduli (summarily denoted by $Z$ ) and the gauge bundle moduli (summarily denoted by $C$ ) as arising at the second order in $W$ schematically as $W=W_{\mathrm{CS},(2)} C^{2} \sim$ $\left(Z+\mathcal{O}\left(Z^{2}\right)\right) C^{2}$.

where the gauge bundle is holomorphic. In the best case scenario where $h_{\text {hol }}^{1,2}=0$ one does not need to turn on any harmonic flux to fix the $Z$-moduli, whereas in the more general case where $h_{\text {hol }}^{1,2}>0$ the remaining complex structure moduli can be fixed only by turning on a quantised background flux. For a graphical sketch of the 'cross-structure' of the combined complex structure and gauge bundle moduli space see figure 1.

The second solution of (3.28) implies that the forms $\omega^{\iota}$ are not closed under $\bar{D}_{0}$ and the index $\iota$ ranges over an infinite set of values. Hence $C_{\iota}$ are not flat directions but correspond to massive deformations, namely the Kaluza-Klein modes. We can then easily realise that $W_{\mathrm{CS},(2)}^{\iota \sigma}$ gives the mass matrix for these Kaluza-Klein modes.

- Focusing only on the massless modes, at quadratic order $F_{C_{i}}=0$ implies:

$$
W_{\mathrm{CS},(3)}^{i j k} C_{j} C_{k}=0 \quad \forall i
$$

showing that a possible obstruction to the presence of gauge bundle moduli can arise if the Yukawa couplings are different from zero, i.e. $W_{\mathrm{CS},(3)}^{i j k} \neq 0 \forall i$. We stress again the fact that $W_{\mathrm{CS},(3)}^{i j k}$ is a function of the $Z$-moduli, and so even if the equation $\bar{D}_{0} \omega^{i}(Z)=0$ (or the flux stabilisation) gives a solution $Z=Z_{*}$ such that $N\left(Z_{*}\right) \neq 0$, one could still fix all the $C$-moduli if $W_{\mathrm{CS},(3)}^{i j k}\left(Z_{*}\right) \neq 0 \forall i$.

Having motivated both the background gauge flux and the nature of the leading deformation we can now work with an arbitrary deformation by separating the set $\left\{C_{I}\right\}=$ 
$\left\{C_{i}^{(0)}\right\} \oplus\left\{C_{\iota}^{\mathrm{KK}}\right\}$ with the first set being the massless modes and the second the KaluzaKlein modes. This corresponds to splitting the set of 1 -forms as $\left\{\omega^{I}\right\}=\left\{\omega_{(0)}^{i}\right\} \oplus\left\{\omega_{\mathrm{KK}}^{\iota}\right\}$ where $\bar{D}_{0} \omega_{(0)}^{i}=0$ while $\bar{D}_{0} \omega_{\mathrm{KK}}^{\iota} \neq 0$. Then under the condition $F_{0,(0,2)}=0$, the F-term equations (3.27) take the form:

$$
0=F_{C_{i}^{(0)}}=2 W_{\mathrm{CS},(3)}^{i k l} C_{k}^{(0)} C_{l}^{(0)}+4 W_{\mathrm{CS},(3)}^{i k \lambda} C_{k}^{(0)} C_{\lambda}^{\mathrm{KK}}+2 W_{\mathrm{CS},(3)}^{i \sigma \lambda} C_{\sigma}^{\mathrm{KK}} C_{\lambda}^{\mathrm{KK}},
$$

and:

$$
0=F_{C_{\iota}^{\mathrm{KK}}}=2 W_{\mathrm{CS},(2)}^{\iota \sigma} C_{\sigma}^{\mathrm{KK}}+2 W_{\mathrm{CS},(3)}^{\iota k l} C_{k}^{(0)} C_{l}^{(0)}+4 W_{\mathrm{CS},(3)}^{\iota k \lambda} C_{k}^{(0)} C_{\lambda}^{\mathrm{KK}}+2 W_{\mathrm{CS},(3)}^{\iota \sigma \lambda} C_{\sigma}^{\mathrm{KK}} C_{\lambda}^{\mathrm{KK}}
$$

Note that $W_{\mathrm{CS},(2)}^{\iota \sigma} \equiv M_{\mathrm{KK}}^{\iota \sigma}$ is the mass matrix for the Kaluza-Klein modes which by definition is non-singular. So eq. (3.31) can be solved for the massive modes in terms of the massless modes giving a relation of the form:

$$
C_{\sigma}^{\mathrm{KK}}=\left[M_{\mathrm{KK}}\right]_{\sigma \lambda}^{-1} W_{\mathrm{CS},(3)}^{\lambda m n} C_{m}^{(0)} C_{n}^{(0)}+O\left(C_{0}^{3}\right) .
$$

Using this in (3.30) we get the massless field equation which is the generalisation of (3.29) for arbitrarily large deformations of the background gauge bundle:

$$
2 W_{\mathrm{CS},(3)}^{i k l} C_{k}^{(0)} C_{l}^{(0)}+O\left(C_{(0)}^{3}\right)=0 .
$$

These field equations always admit the solution $C_{k}^{(0)}=0$ for all gauge bundle moduli which leaves the complex structure moduli unfixed in the absence of harmonic quantised flux. Moreover, this solution remains valid even in the presence of non-zero $W$ since the additional term in $D_{C} W$ is proportional to $C$. However one could also have solutions with non-zero VEVs for the $C$-moduli which could be obtained by cancelling field-dependent FI-terms associated with anomalous $U(1)$ factors. By the cross structure of the combined moduli space [33-35], this in turn implies stabilisation of at most $h^{1,2}-h_{\text {hol }}^{1,2}$ complex structure moduli. This situation is particularly relevant for the case of heterotic orbifold compactifications which often have only a few untwisted $Z$-moduli. In this case it seems possible to stabilise all gauge bundle moduli and the small total number of untwisted complex structure moduli using only higher-order terms in (3.33) and a sufficient number of D-terms from anomalous $\mathrm{U}(1)$ factors [4-8].

In the rest of the paper we will focus on the generic situation where this stabilisation procedure fixes all the gauge bundle moduli and some, but not all, complex structure moduli, so that $h_{\text {hol }}^{1,2}>0 Z$-moduli are still left flat. Furthermore, even if $h_{\text {hol }}^{1,2}=0$, it could still be that some $C$-moduli are fixed at zero VEV, implying that the complex structure moduli could still be flat (see figure 1).

\subsection{Corrections beyond tree-level}

Given that the remaining $h_{\text {hol }}^{1,2}>0 Z$-moduli cannot be fixed at tree-level by using quantised fluxes (since $\left|W_{0}\right| \neq 0$ would induce a runaway for both $s$ and $\mathcal{V}$ ), let us focus on perturbative and non-perturbative corrections to the scalar potential. We shall proceed in 
two steps, showing first how to fix the complex structure moduli and the dilaton by the inclusion of an $S$-dependent gaugino condensate, and then explaining how to stabilise the Kähler moduli by an interplay of world-sheet instantons and threshold corrections to the gauge kinetic function. For the time being, we shall neglect perturbative corrections to the Kähler potential (either $\alpha^{\prime}$ or $g_{s}$ ) since these generically break supersymmetry, and so we shall include them only in section 4 where we shall study supersymmetry breaking vacua.

\subsubsection{Step 1: $Z$ and $S$ stabilisation by gaugino condensation}

Let us add a single $S$-dependent gaugino condensate to the superpotential and determine how this term modifies the tree-level picture:

$$
W=W_{\text {flux }}+W_{\text {gc }}=\int_{X} H \wedge \Omega+A(Z) e^{-\lambda S} .
$$

The Kähler-covariant derivatives now become:

$$
\begin{aligned}
D_{Z^{\alpha}} W & =\mathrm{i} b_{\alpha}(Z)+e^{-\lambda S}\left[\partial_{\alpha} A(Z)-k_{\alpha}(Z, \bar{Z}) A(Z)\right], \\
D_{S} W & =-\frac{1}{2 s}\left[\mathrm{i} a(Z)+(2 \lambda s+1) A(Z) e^{-\lambda S}\right], \\
D_{T_{i}} W & =-\frac{\mathrm{i} a(Z)+A(Z) e^{-\lambda S}}{4 \mathcal{V}} k_{i j k} t_{j} t_{k} .
\end{aligned}
$$

The potential is again of the no-scale type (i.e. given by the first equality of (3.20)). At the minimum the complex structure moduli will be frozen at the solution to:

$$
D_{Z^{\alpha}} W=0 \quad \Leftrightarrow \quad \mathrm{i} b_{\alpha}(Z)=e^{-\lambda S}\left[k_{\alpha}(Z, \bar{Z}) A(Z)-\partial_{\alpha} A(Z)\right],
$$

and now the dilaton is not forced anymore to run-away to infinity:

$$
D_{S} W=0 \quad \Leftrightarrow \quad W_{0} \equiv \mathrm{i} a(Z)=-(2 \lambda s+1) A(Z) e^{-\lambda S} .
$$

The potential for the Kähler moduli is flat, resulting in a Minkowski vacuum with broken supersymmetry since substituting (3.39) into (3.37) one finds:

$$
D_{T_{i}} W=-\left(\frac{2 \lambda s}{2 \lambda s+1}\right) \frac{W_{0}}{4 \mathcal{V}} k_{i j k} t_{j} t_{k} .
$$

The previous expression for $W_{0} \neq 0$, finite volume and $t_{i}>1 \forall i$, gives $D_{T_{i}} W \neq 0$ for a generic point in moduli space.

Let us comment now on the possibility to satisfy (3.39) at the physical point $\langle s\rangle \simeq 2$ that corresponds to $\alpha_{G U T}^{-1} \simeq 25$. Setting $A=1$ and $\lambda=8 \pi^{2} / N$ where $N$ is the rank of the $\mathrm{SU}(N)$ condensing gauge group, we have (fixing the axion $a$ at $\lambda\langle a\rangle=\pi$ ):

$$
W_{0}=\left(\frac{16 \pi^{2}\langle s\rangle}{N}+1\right) e^{-\frac{8 \pi^{2}}{N}\langle s\rangle}
$$

As an illustrative example, for $\langle s\rangle \simeq 2$ and $N=5$, the previous expression would give $W_{0} \simeq 10^{-12}$, which for $\mathcal{V} \simeq 20$ corresponds to a gravitino mass of the order $m_{3 / 2}=$ 
$W_{0} /(\sqrt{2 s \mathcal{V}}) \simeq 330 \mathrm{TeV}$. On the other hand, for $N=30$ (as in the case of $E_{8}$ ), one would obtain $W_{0} \simeq 0.06$ corresponding to a GUT-scale gravitino mass: $m_{3 / 2} \simeq 10^{16} \mathrm{GeV}$. Due to the absence of Ramond-Ramond fluxes, there is in general no freedom to tune the heterotic flux superpotential $W_{0}$ to values much smaller than unity, implying that heterotic CY compactifications generically predict a gravitino mass close to the GUT scale. As we already pointed out, low-energy supersymmetry could instead be obtained in the particular cases when $h_{\text {hol }}^{1,2}=0$ so that one does not need to turn on $W_{0} \neq 0$ to fix all the $Z$-moduli, in orbifold constructions or in compactifications on non-complex manifolds.

A possible way to obtain fractional values of $W_{0}$ of order $0.1-0.01$ has been described in [17] where the authors considered a trivial $B_{2}$ field and a rigid 3-cycle $\Sigma_{3}$ such that the integral of $H$ over $\Sigma_{3}$ (ignoring the contribution from the spin connection):

$$
\int_{\Sigma_{3}} H \simeq-\int_{\Sigma_{3}} \operatorname{CS}(A)
$$

gives rise to a fractional flux. ${ }^{11}$ Stabilisation of all complex structure moduli would then require scanning the three-form flux over all cycles to search for VEVs $\left\langle Z_{\alpha}\right\rangle$ such that the overall $(0,3)$-contribution to the superpotential (3.13) is of the order of the fractional Chern-Simons contribution or smaller. ${ }^{12}$

\subsubsection{Step 2: $T$ stabilisation by worldsheet instantons and threshold effects}

The Kähler moduli can develop a potential either by loop corrections to the gauge kinetic function or via worldsheet instantons. Let us start considering the case with just threshold effects.

Threshold effects: The potential generated by gaugino condensation takes the form:

$$
W_{\mathrm{gc}}=A(Z) e^{-\lambda\left(S-\frac{\beta_{i}}{2} T_{i}\right)}
$$

lifting the $T$-moduli and modifying (3.40) into:

$$
D_{T_{i}} W=-\frac{\lambda W_{0}}{2(2 \lambda s+1)}\left[\beta_{i}+\frac{s}{\mathcal{V}} k_{i j k} t_{j} t_{k}\right]=0 \quad \Leftrightarrow \quad \beta_{i}=-\frac{s}{\mathcal{V}} k_{i j k} t_{j} t_{k} .
$$

This result, in turn, gives:

$$
\operatorname{Re}\left(f_{\text {hid }}^{1-\text { loop }}\right)=-\frac{\beta_{i}}{2} t_{i}=\frac{s}{2 \mathcal{V}} k_{i j k} t_{i} t_{j} t_{k}=3 s=3 \operatorname{Re}\left(f_{\text {hid }}^{\text {tree }}\right),
$$

\footnotetext{
${ }^{11}$ Note that these flux quanta are well-defined quantities even if $H$ is not closed since a rigid homology class admits just one representative

${ }^{12}$ For the purpose of an explicit demonstration of such vacua one may rely on CYs arising in Greene-Plesser pairs of manifolds related by mirror symmetry [52-54]. CY mirror pairs arising from the Greene-Plesser construction have their complex structure moduli space partitioned by a typically large discrete symmetry $\Gamma$ into an invariant subspace and its complement. One can then show that the periods of the invariant subspace depend at higher-order non-trivially on all the $\Gamma$-non-invariant complex structure moduli. If the $\Gamma$-invariant subspace is of low dimensionality (as is the case e.g. of the $\mathrm{CY} \mathbb{C P}_{11169}^{4}[18]$ as discussed in $[55,56])$, then turning on the relatively few fluxes on the invariant subspace is enough to stabilise all complex structure moduli at an isolated minimum $[55,56]$. On such a CY manifold one can therefore stabilise all $Z$-moduli by just turning a few fractional Chern-Simons $(0,3)$-type fluxes on the cycles of the invariant subspace, which can serve to demonstrate the existence of such vacua.
} 
implying that perturbation theory in the hidden sector is not under control since the oneloop contribution is bigger than the tree-level one. Moreover the gauge kinetic function of the visible sector becomes negative:

$$
\operatorname{Re}\left(f_{\text {vis }}\right)=g_{\text {vis }}^{-2}=s+\frac{\beta_{i}}{2} t_{i}=-2 s<0,
$$

meaning that the positive tree-level contribution is driven to negative values by threshold effects. Actually, before becoming negative, $g_{\text {vis }}^{-2}$ will vanish corresponding to a strong coupling transition whose understanding is not very clear [17]. Note that we neglected D-terms since, due to the relation (2.31), if present, they would also cause the same problems. Let us see now how these control issues can be addressed by including worldsheet instantons [19].

Threshold effects and worldsheet instantons: The new total non-perturbative superpotential reads:

$$
W_{\mathrm{np}}=A(Z) e^{-\lambda\left(S-\frac{\beta_{i}}{2} T_{i}\right)}+B(Z) e^{-\mu T_{*}},
$$

where we included the contribution of a single worldsheet instanton dependent on $T_{*}$. In general, one could have more non-perturbative $\alpha^{\prime}$ contributions, but we shall here show that just one worldsheet instanton is enough to overcome the previous problems. The new Kähler covariant derivatives become:

$$
\begin{aligned}
D_{Z^{\alpha}} W & =\mathrm{i} b_{\alpha}(Z)+W_{\mathrm{gc}}\left[\frac{\partial_{\alpha} A(Z)}{A(Z)}-k_{\alpha}(Z, \bar{Z})\right]+W_{\mathrm{wi}}\left[\frac{\partial_{\alpha} B(Z)}{B(Z)}-k_{\alpha}(Z, \bar{Z})\right], \\
D_{S} W & =-\frac{1}{2 s}\left[W_{0}+(2 \lambda s+1) W_{\mathrm{gc}}+W_{\mathrm{wi}}\right] \\
D_{T_{p}} W & =\lambda \frac{\beta_{p}}{2} W_{\mathrm{gc}}-\frac{W_{0}+W_{\mathrm{gc}}+W_{\mathrm{wi}}}{4 \mathcal{V}} k_{p j k} t_{j} t_{k} \quad p \neq * \\
D_{T_{*}} W & =\lambda \frac{\beta_{*}}{2} W_{\mathrm{gc}}-\mu W_{\mathrm{wi}}-\frac{W_{0}+W_{\mathrm{gc}}+W_{\mathrm{wi}}}{4 \mathcal{V}} k_{* j k} t_{j} t_{k}
\end{aligned}
$$

The solutions describing supersymmetric vacua with vanishing F-terms are:

$$
\begin{aligned}
\mathrm{i} b_{\alpha}(Z) & =W_{\mathrm{gc}}\left[k_{\alpha}(Z, \bar{Z})-\frac{\partial_{\alpha} A(Z)}{A(Z)}\right]+W_{\mathrm{wi}}\left[k_{\alpha}(Z, \bar{Z})-\frac{\partial_{\alpha} B(Z)}{B(Z)}\right], \\
W_{0} & =-(2 \lambda s+1) W_{\mathrm{gc}}-W_{\mathrm{wi}}, \\
\beta_{p} & =-\frac{s}{\mathcal{V}} k_{p j k} t_{j} t_{k} \quad p \neq *, \\
\beta_{*} & =-\frac{s}{\mathcal{V}} k_{* j k} t_{j} t_{k}+2 R, \quad R \equiv \frac{\mu W_{\mathrm{wi}}}{\lambda W_{\mathrm{gc}}} .
\end{aligned}
$$

It is important to note that the total superpotential $W=W_{0}+W_{\mathrm{gc}}+W_{\mathrm{wi}} \neq 0$. Indeed if this were zero the dilaton would not be stabilised (see (3.53)). This of course means that the supersymmetric vacua are AdS in contrast to Strominger's classical analysis [20].

The hidden and visible sector gauge kinetic functions now improve their behaviour since they look like:

$$
\operatorname{Re}\left(f_{\text {hid }}^{1-\text { loop }}\right)=-\frac{\beta_{i}}{2} t_{i}=3 s-R t_{*}=3 \operatorname{Re}\left(f_{\text {hid }}^{\text {tree }}\right)-R t_{*}
$$


and:

$$
\operatorname{Re}\left(f_{\text {vis }}\right)=-2 s+R t_{*} .
$$

Thus there is a regime where the hidden sector is weakly coupled and the real part of the gauge kinetic function of the visible sector (as well as that of the hidden sector) stays positive for:

$$
2 s \ll R t_{*} \ll 4 s,
$$

which points towards values $R t_{*} \simeq 3 \mathrm{~s}$. In fact, in this regime, not only $\operatorname{Re}\left(f_{\mathrm{vis}}\right)>0$ and $\operatorname{Re}\left(f_{\text {hid }}\right)>0$, but also:

$$
\left|\frac{\operatorname{Re}\left(f_{\text {hid }}^{1-\text { loop }}\right)}{\operatorname{Re}\left(f_{\text {hid }}^{\text {tree }}\right)}\right|=\left|\frac{\operatorname{Re}\left(f_{\text {vis }}^{1-\text { loop }}\right)}{\operatorname{Re}\left(f_{\text {vis }}^{\text {tree }}\right)}\right|=\left|3-\frac{R t_{*}}{s}\right| \ll 1 .
$$

\subsubsection{Tuning the Calabi-Yau condition}

As pointed out in [18], in the absence of worldsheet instantons and for $\partial_{\alpha} A(Z)=0$, eq. (3.48) reduces to:

$$
\mathrm{i} b_{\alpha}=W_{\mathrm{gc}} k_{\alpha}(Z, \bar{Z}) \neq 0 .
$$

This induces a $(2,1)$-component of $H$ (harmonic) that should vanish according to Strominger's analysis [20]. However from (3.52), one may speculate that the CY condition can be preserved by envisaging a situation where one tunes the flux quanta such that $b_{\alpha}=0$ $\forall \alpha=1, \ldots, h_{\text {hol }}^{1,2}$ corresponding to $H^{2,1}=0$. The complex structure moduli would then be fixed by:

$$
D_{Z^{\alpha}} W=0 \quad \Leftrightarrow \quad \frac{W_{\mathrm{wi}}}{W_{\mathrm{gc}}}=-\frac{1-\frac{\partial_{\alpha} A(Z)}{A(Z) k_{\alpha}(Z, \bar{Z})}}{1-\frac{\partial_{\alpha} B(Z)}{B(Z) k_{\alpha}(Z, \bar{Z})}} .
$$

However now we have $4 h_{\text {hol }}^{1,2}$ real equations determining $2 h_{\text {hol }}^{1,2}$ real complex structure moduli. Obviously the system has no solution unless we scan over the integer fluxes. However there are only $2 h^{1,2}+2$ integer fluxes. Thus we have only the freedom to scan over $Q=2\left(h^{1,2}-h_{\text {hol }}^{1,2}+1\right)$ integers while all $2 h_{\text {hol }}^{1,2}$ real complex structure moduli as well as all but $Q$ of the integers (i.e. $2 h_{\text {hol }}^{1,2}$ of them) must emerge as solutions to these non-linear equations. Thus we do not think that it is possible to have $b_{\alpha}=0$ in the presence of these non-perturbative terms. However, this condition emerges only on demanding a supersymmetric solution to the classical 10D equations, and so our 4D analysis cannot be expected to satisfy these classical conditions once non-perturbative effects are included.

\subsection{Flux vacua counting}

Let us clarify here a crucial difference between type IIB and heterotic string theory regarding complex structure stabilisation with three-form flux. The F-term equations (3.48) comprise $2 h^{1,2}$ conditions for $2 h^{1,2}$ real variables (setting now $h_{\text {hol }}^{1,2}=h^{1,2}$ for ease of comparison with type IIB). A non-trivial $H$-flux yields exactly $2 h^{1,2}$ independent flux quanta (up to the two related to the overall scaling of $\Omega(Z)$ ) generically supplying the non-linear system of $h^{1,2}$ complex F-term conditions for the $2 h^{1,2}$ complex structure moduli. However, the 
existence of a finite number of isolated solutions for such non-linear systems with as many equations as variables (rendering the system 'well behaved') is not guaranteed. One expects therefore that most of the available freedom of choice among the $2 h^{1,2} H$-fluxes is used up to find a relatively small number of isolated solutions for the complex structure moduli where all of them sit safely in the regime of large complex structure. Generically, this precludes the possibility of using the $H$-flux discretuum for tuning a very small VEV of $W_{\text {flux }}$.

Note that this is different in the type IIB context. There, the availability of RR threeform flux $F_{3}$ supplies an additional set of $2 h^{2,1}$ fluxes for an overall discretuum made up from $4 h^{1,2}$ fluxes. We have therefore an additional set of $2 h^{1,2}$ discrete parameters available for tuning $W_{\text {flux }}$ while keeping a given well-behaved complex structure moduli vacuum. Consequently, after having used $2 h^{1,2}$ flux parameters to construct a viable complex structure vacuum, we can use the additional $2 h^{1,2}$ flux quanta to construct a 'discrete $2 h^{1,2}$-parameter family' of complex structure vacua, which allows for exponential tuning of $W_{\text {flux }}$.

Finally we note that in the heterotic case the unavailability of any additional freedom in the flux choice after fixing the $Z$-moduli, means that we have to depend on the far more restricted choices that are available in the solution space of the complex structure moduli. As mentioned before, one needs to scan over the $H$ flux integers in order to find $2 h^{1,2}$ acceptable (i.e. in the geometric regime) real solutions to the $2 h^{1,2}$ non-linear equations $D_{Z^{\alpha}} W=0$. The size of the solution set that we get is likely to be much smaller than the size of the original set of flux integers. Thus even if we had started with, let us say, $h^{1,2}=\mathcal{O}(100)$ and let each flux scan over 1 to 10, the number of acceptable fluxes are likely to be far smaller than what is required to tune the cosmological constant. It should also be emphasised here that the only source of tuning that is available after all the low-energy contributions to the vacuum energy are included, has to come from these fluxes.

\section{Supersymmetry breaking vacua}

In this section we shall show the existence of new Minkowski vacua with spontaneous supersymmetry breaking along the Kähler moduli directions. The strategy is to perform moduli stabilisation in two steps as follows:

- Step 1: Fix at leading order some of the moduli supersymmetrically (all the $h_{\text {hol }}^{1,2}>0$ complex structure moduli, the dilaton and some Kähler moduli) at a high scale.

- Step 2: Stabilise the remaining light moduli at a lower scale breaking supersymmetry mainly along the Kähler directions by the inclusion of $\alpha^{\prime}$ corrections to the Kähler potential in a way similar to type IIB.

In subsection 4.1 we shall consider the contributions to the scalar potential generated by fluxes, non-perturbative effects and threshold corrections showing that there exist no supersymmetry breaking minimum which lies in the regime of validity of the effective field theory. However, in subsection 4.2 we shall describe how this situation improves by the inclusion of $\alpha^{\prime}$ corrections to the Kähler potential which yield trustworthy Minkowski vacua 
(see subsection 4.3) where supersymmetry is spontaneously broken by the F-terms of the Kähler moduli. ${ }^{13}$ In subsection 4.4 we shall explain what is the rôle played by D-terms in our stabilisation procedure. Let us finally stress that this new procedure to obtain supersymmetry breaking vacua is completely orthogonal to the way the complex structure moduli are fixed, and so our results apply also to the case with $h_{\mathrm{hol}}^{1,2}=0$ where there is no need to turn on quantised background fluxes to fix the $Z$-moduli.

\subsection{Fluxes, non-perturbative effects and threshold corrections}

In this section we shall derive the general expression for the scalar potential including fluxes, non-perturbative effects (both gaugino condensation and world-sheet instantons) and threshold corrections for a CY three-fold whose volume is given by:

$$
\mathcal{V}=k_{b} t_{b}^{3}-k_{s} t_{s}^{3}
$$

The superpotential and the Kähler potential look like (neglecting a possible $Z$-dependence of $A$ and $B$ and setting for simplicity $\beta_{s}=0$ ):

$$
\begin{aligned}
W & =W_{\text {flux }}(Z)+A e^{-\lambda\left(S-\frac{\beta_{b}}{2} T_{b}\right)}+B e^{-\mu T_{s}}, \\
K & =-\ln \mathcal{V}-\ln (S+\bar{S})+K_{\mathrm{cs}}(Z, \bar{Z}) .
\end{aligned}
$$

Performing the following field redefinition:

$$
\Phi \equiv S-\frac{\beta_{b}}{2} T_{b}
$$

$W$ and $K$ take the form:

$$
\begin{aligned}
W & =W_{\text {flux }}(Z)+A e^{-\lambda \Phi}+B e^{-\mu T_{s}}, \\
K & =-\ln \mathcal{V}-\ln \left[\Phi+\bar{\Phi}+\frac{\beta_{b}}{2}\left(T_{b}+\bar{T}_{b}\right)\right]+K_{\mathrm{cs}}(Z, \bar{Z}) .
\end{aligned}
$$

\subsubsection{Derivation of the F-term potential}

The F-term scalar potential turns out to be:

$$
\begin{aligned}
V= & e^{K}\left[\sum_{Z} K^{\alpha \bar{\beta}} D_{\alpha} W D_{\bar{\beta}} \bar{W}+K^{\Phi \bar{\Phi}} D_{\Phi} W D_{\bar{\Phi}} \bar{W}\right. \\
& +\left(K^{\Phi \bar{T}_{b}} K_{\bar{T}_{b}}+K^{\Phi \bar{T}_{s}} K_{\bar{T}_{s}}\right)\left(\bar{W} D_{\Phi} W+W D_{\bar{\Phi}} \bar{W}\right) \\
& +K^{\Phi \bar{T}_{s}} \partial_{\bar{T}_{s}} \bar{W} D_{\Phi} W+K^{T_{s}} \bar{\Phi} \partial_{T_{s}} W D_{\bar{\Phi}} \bar{W} \\
& +|W|^{2}\left(\sum_{T} K^{i \bar{j}} K_{i} K_{\bar{j}}-3\right) \\
& +\left(K^{T_{s} \bar{T}_{b}} K_{\bar{T}_{b}}+K^{T_{s} \bar{T}_{s}} K_{\bar{T}_{s}}\right)\left(\bar{W} \partial_{T_{s}} W+W \partial_{\bar{T}_{s}} \bar{W}\right) \\
& \left.+K^{T_{s} \bar{T}_{s}} \partial_{T_{s}} W \partial_{\bar{T}_{s}} \bar{W}\right] .
\end{aligned}
$$

\footnotetext{
${ }^{13}$ See [57] for another attempt to fix the heterotic moduli via the inclusion of $\alpha^{\prime}$ effects.
} 
Let us consider the limit:

$$
\left|\operatorname{Re}\left(f_{\text {hid }}^{1-\text { loop }}\right)\right| \ll \operatorname{Re}\left(f_{\text {hid }}^{\text {tree }}\right) \Leftrightarrow \frac{\beta_{b}}{2} t_{b} \ll s,
$$

which implies (defining $\Phi=\phi+\mathrm{i} \psi$ ):

$$
\epsilon_{\phi} \equiv \frac{\beta_{b} t_{b}}{2 \phi}=\frac{\beta_{b} t_{b}}{2\left(s-\frac{\beta_{b}}{2} t_{b}\right)}=-\frac{1}{1-\frac{2 s}{\beta_{b} t_{b}}} \simeq \frac{\beta_{b} t_{b}}{2 s} \ll 1,
$$

together with:

$$
t_{b} \sim \mathcal{O}(10)>t_{s} \sim \mathcal{O}(1) \quad \Rightarrow \quad \epsilon_{s} \equiv \frac{k_{s} t_{s}^{3}}{k_{b} t_{b}^{3}} \ll 1 .
$$

We can then expand the relevant terms as:

$$
\begin{gathered}
K^{\Phi \bar{\Phi}=4 \phi^{2}}\left(1+2 \epsilon_{\phi}+\frac{4 \epsilon_{\phi}^{2}}{3}+\frac{\epsilon_{s} \epsilon_{\phi}^{2}}{6}\right), \quad K^{\Phi \bar{T}_{s}}=K^{T_{s} \bar{\Phi}}=-2 \epsilon_{\phi} \phi t_{s}, \\
K^{\Phi \bar{T}_{b}} K_{\bar{T}_{b}}+K^{\Phi \bar{T}_{s}} K_{\bar{T}_{s}}=\frac{2 \epsilon_{\phi} \phi}{1+\epsilon_{\phi}}\left(1+\frac{4 \epsilon_{\phi}}{3}+\frac{\epsilon_{s} \epsilon_{\phi}}{6}\right) .
\end{gathered}
$$

The no-scale structure gets broken by loop effects:

$$
\sum_{T} K^{i \bar{j}} K_{i} K_{\bar{j}}-3=\frac{2 \epsilon_{\phi}}{\left(1+\epsilon_{\phi}\right)^{2}}\left(1+\frac{7 \epsilon_{\phi}}{6}+\frac{\epsilon_{\phi} \epsilon_{s}}{12}\right) .
$$

Note that one correctly recovers the no-scale cancellation for $\beta_{b}=0 \Leftrightarrow \epsilon_{\phi}=0$. Other relevant terms are:

$$
K^{T_{s} \bar{T}_{b}} K_{\bar{T}_{b}}+K^{T_{s} \bar{T}_{s}} K_{\bar{T}_{s}}=-2 t_{s}\left(\frac{1+3 \epsilon_{\phi} / 2}{1+\epsilon_{\phi}}\right), \quad K^{T_{s} \bar{T}_{s}}=\frac{2 t_{s}^{2}}{3 \epsilon_{s}}\left(1+2 \epsilon_{s}\right) .
$$

We shall look for minima in the region $\mathcal{V} \sim W_{\text {flux }} e^{\mu t_{s}}$ implying that $W_{\text {wi }} \sim \epsilon_{s} W_{\text {flux }} \ll$ $W_{\text {flux }} \sim W_{\text {gc }}$. The relevant derivatives scale as:

$$
\partial_{Z^{\alpha}} W \sim W_{\text {flux }}, \quad \partial_{\Phi} W \sim W_{\text {gc }} \sim W_{\text {flux }}, \quad \partial_{T_{s}} W \sim W_{\text {wi }} \sim \epsilon_{s} W_{\text {flux }} .
$$

Therefore the F-term scalar potential can be expanded in the small parameters $\epsilon_{\phi}$ and $\epsilon_{s}$ as:

$$
V=V_{0}+\epsilon V_{1}+\epsilon^{2} V_{2}+\ldots
$$

where (defining $\hat{W}=W_{\text {flux }}+W_{\text {gc }}$ ):

$$
V_{0}=e^{K}\left(\sum_{Z} K^{\alpha \bar{\beta}} D_{\alpha} \hat{W} D_{\bar{\beta}} \overline{\hat{W}}+4 \phi^{2} D_{\Phi} \hat{W} D_{\bar{\Phi}} \overline{\hat{W}}\right) \sim \mathcal{O}\left(e^{K}\left|W_{\text {flux }}\right|^{2}\right),
$$

and:

$$
\begin{aligned}
\epsilon V_{1}= & e^{K}\left[\sum_{Z} K^{\alpha \bar{\beta}}\left(D_{\alpha} \hat{W} D_{\bar{\beta}} \bar{W}_{\mathrm{wi}}+D_{\alpha} W_{\mathrm{wi}} D_{\bar{\beta}} \overline{\hat{W}}\right)+4 \phi^{2}\left(D_{\Phi} \hat{W} D_{\bar{\Phi}} \bar{W}_{\mathrm{wi}}+D_{\Phi} W_{\mathrm{wi}} D_{\bar{\Phi}} \overline{\hat{W}}\right)\right. \\
& +8 \epsilon_{\phi} \phi^{2} D_{\Phi} \hat{W} D_{\bar{\Phi}} \overline{\hat{W}}+2 \epsilon_{\phi} \phi\left(\overline{\hat{W}} D_{\Phi} \hat{W}+\hat{W} D_{\bar{\Phi}} \overline{\hat{W}}\right) \\
& \left.+2|\hat{W}|^{2} \epsilon_{\phi}-2 t_{s}\left(\overline{\hat{W}} \partial_{T_{s}} W+\hat{W} \partial_{\bar{T}_{s}} \bar{W}\right)+\frac{2 t_{s}^{2}}{3 \epsilon_{s}} \partial_{T_{s}} W \partial_{\bar{T}_{s}} \bar{W}\right] \sim \mathcal{O}\left(\epsilon e^{K}\left|W_{\text {flux }}\right|^{2}\right),
\end{aligned}
$$


and:

$$
\begin{aligned}
\epsilon^{2} V_{2}= & e^{K}\left[\sum_{Z} K^{\alpha \bar{\beta}} D_{\alpha} W_{\mathrm{wi}} D_{\bar{\beta}} \bar{W}_{\mathrm{wi}}+4 \phi^{2} D_{\Phi} W_{\mathrm{wi}} D_{\bar{\Phi}} \bar{W}_{\mathrm{wi}}+8 \epsilon_{\phi} \phi^{2}\left(D_{\Phi} \hat{W} D_{\bar{\Phi}} \bar{W}_{\mathrm{wi}}+\text { h.c. }\right)\right. \\
& +\frac{16}{3} \epsilon_{\phi}^{2} \phi^{2} D_{\Phi} \hat{W} D_{\bar{\Phi}} \overline{\hat{W}}+2 \epsilon_{\phi} \phi\left(\overline{\hat{W}} D_{\Phi} W_{\mathrm{wi}}+W_{\mathrm{wi}} D_{\bar{\Phi}} \overline{\hat{W}}+\text { h.c. }\right)+\frac{2 \epsilon_{\phi}^{2} \phi}{3}\left(\overline{\hat{W}} D_{\Phi} \hat{W}+\text { h.c. }\right) \\
& -2 \epsilon_{\phi} \phi t_{s}\left(\partial_{\overline{T_{s}}} \bar{W} D_{\Phi} \hat{W}+\text { h.c. }\right)+2 \epsilon_{\phi}\left(\hat{W} \bar{W}_{\mathrm{wi}}+\text { h.c. }\right)-\frac{5 \epsilon_{\phi}^{2}}{3}|\hat{W}|^{2} \\
& \left.-2 t_{s}\left(\bar{W}_{\mathrm{wi}} \partial_{T_{s}} W+\text { h.c. }\right)-t_{s} \epsilon_{\phi}\left(\overline{\hat{W}} \partial_{T_{s}} W+\text { h.c. }\right)+\frac{4 t_{s}^{2}}{3} \partial_{T_{s}} W \partial_{\bar{T}_{s}} \bar{W}\right] \sim \mathcal{O}\left(\epsilon^{2} e^{K}\left|W_{\text {flux }}\right|^{2}\right) .
\end{aligned}
$$

\subsubsection{Moduli stabilisation}

Let us perform moduli stabilisation in two steps.

Step 1: We stabilise the $\Phi$ and $Z$-moduli by imposing $D_{Z^{\alpha}} \hat{W}=D_{\Phi} \hat{W}=0$ thus minimising the leading order term in the potential. We then substitute this solution in the scalar potential obtaining $V_{0}=0$ whereas the other contributions take the form:

$$
\epsilon V_{1}=e^{K}\left[2|\hat{W}|^{2} \epsilon_{\phi}-2 t_{s}\left(\overline{\hat{W}} \partial_{T_{s}} W+\hat{W} \partial_{\bar{T}_{s}} \bar{W}\right)+\frac{2 t_{s}^{2}}{3 \epsilon_{s}} \partial_{T_{s}} W \partial_{\bar{T}_{s}} \bar{W}\right]
$$

and:

$$
\begin{aligned}
\epsilon^{2} V_{2}= & e^{K}\left[\sum_{Z} K^{\alpha \bar{\beta}} D_{\alpha} W_{\mathrm{wi}} D_{\bar{\beta}} \bar{W}_{\mathrm{wi}}+4 \phi^{2} D_{\Phi} W_{\mathrm{wi}} D_{\bar{\Phi}} \bar{W}_{\mathrm{wi}}\right. \\
& +2 \epsilon_{\phi} \phi\left(\overline{\hat{W}} D_{\Phi} W_{\mathrm{wi}}+\text { h.c. }\right)+2 \epsilon_{\phi}\left(\hat{W} \bar{W}_{\mathrm{wi}}+\text { h.c. }\right)-\frac{5 \epsilon_{\phi}^{2}}{3}|\hat{W}|^{2} \\
& \left.-2 t_{s}\left(\bar{W}_{\mathrm{wi}} \partial_{T_{s}} W+\text { h.c. }\right)-t_{s} \epsilon_{\phi}\left(\overline{\hat{W}} \partial_{T_{s}} W+\text { h.c. }\right)+\frac{4 t_{s}^{2}}{3} \partial_{T_{s}} W \partial_{\bar{T}_{s}} \bar{W}\right] .
\end{aligned}
$$

Step 2: We stabilise the $T$-moduli at order $\mathcal{O}(\epsilon)$ breaking supersymmetry. Writing $T_{s}=t_{s}+\mathrm{i} a_{s}, W_{0}^{\text {eff }}=\left|W_{0}^{\text {eff }}\right| e^{\mathrm{i} \theta_{W}}$ and $B=|B| e^{\mathrm{i} \theta_{B}}$, and setting $e^{\left\langle K_{\mathrm{cs}}\right\rangle}=1$, the explicit form of the scalar potential at $\mathcal{O}(\epsilon)$ is:

$$
V=\left[\frac{A_{1}}{\mathcal{V}^{2 / 3}}+\frac{\left|A_{2}\right|}{\left|W_{0}^{\text {eff }}\right|} \cos \left(\theta_{B}-\theta_{W}-\mu a_{s}\right) \frac{t_{s} e^{-\mu t_{s}}}{\mathcal{V}}+\frac{\left|A_{3}\right|}{\left|W_{0}^{\text {eff }}\right|^{2}} \frac{e^{-2 \mu t_{s}}}{t_{s}}\right] \frac{\left|W_{0}^{\text {eff }}\right|^{2}}{\langle\phi\rangle},
$$

with:

$$
A_{1} \equiv \frac{\beta_{b}}{2\langle\phi\rangle k_{b}^{1 / 3}}, \quad\left|A_{2}\right| \equiv 2 \mu|B|, \quad\left|A_{3}\right| \equiv \frac{\mu^{2}|B|^{2}}{3 k_{s}},
$$

where we have defined $W_{0}^{\text {eff }} \equiv\langle\hat{W}\rangle=\left\langle W_{\text {flux }}+W_{\text {gc }}\right\rangle$. The axion $a_{s}$ is minimised at $\mu\left\langle a_{s}\right\rangle=\theta_{B}-\theta_{W}-\pi$ so that (4.16) reduces to:

$$
V=\left[\frac{A_{1}}{\mathcal{V}^{2 / 3}}-\frac{\left|A_{2}\right|}{\left|W_{0}^{\text {eff }}\right|} \frac{t_{s} e^{-\mu t_{s}}}{\mathcal{V}}+\frac{\left|A_{3}\right|}{\left|W_{0}^{\text {eff }}\right|^{2}} \frac{e^{-2 \mu t_{s}}}{t_{s}}\right] \frac{\left|W_{0}^{\text {eff }}\right|^{2}}{\langle\phi\rangle} .
$$

Minimising with respect to $t_{s}$ one finds:

$$
\mathcal{V}=\frac{\left|A_{2}\right|\left|W_{0}^{\mathrm{eff}}\right|}{\left|A_{3}\right|} \frac{\left(\mu t_{s}-1\right)}{\left(2 \mu t_{s}+1\right)} t_{s}^{2} e^{\mu t_{s}} \underset{\mu t_{s} \gg 1}{\simeq} \frac{\left|A_{2}\right|\left|W_{0}^{\mathrm{eff}}\right|}{2\left|A_{3}\right|} t_{s}^{2} e^{\mu t_{s}}=\frac{3 k_{s} t_{s}^{2}}{\mu|B|}\left|W_{0}^{\mathrm{eff}}\right| e^{\mu t_{s}},
$$


which implies:

$$
\mu t_{s}=\ln \left(\frac{\mathcal{V}}{\left|\lambda_{0}\right|}\right)-2 \ln t_{s} \underset{t_{s} \sim \mathcal{O}(1)}{\simeq} \ln \left(\frac{\mathcal{V}}{\left|\lambda_{0}\right|}\right) \equiv x(\mathcal{V}) \quad \text { with } \quad\left|\lambda_{0}\right| \equiv \frac{3 k_{s}\left|W_{0}^{\text {eff }}\right|}{\mu|B|}
$$

Note that we can trust our effective field theory when $t_{s} \geq 1$, that is when $x(\mathcal{V}) \geq \mu=2 \pi$. Substituting (4.19) and (4.20) in (4.18), we end up with:

$$
V=\left[A_{1} \mathcal{V}^{4 / 3}-\left|C_{0}\right| x(\mathcal{V})^{3}\right] \frac{\left|W_{0}^{\mathrm{eff}}\right|^{2}}{\langle\phi\rangle \mathcal{V}^{2}}, \quad \text { where } \quad\left|C_{0}\right| \equiv \frac{3 k_{s}}{\mu^{3}} .
$$

The extrema of $V$ are located at:

$$
\frac{\partial V}{\partial \mathcal{V}}=0 \quad \Leftrightarrow \quad A_{1} \mathcal{V}^{4 / 3}=3\left|C_{0}\right| x(\mathcal{V})^{2}\left[x(\mathcal{V})-\frac{3}{2}\right],
$$

showing that $A_{1}$ has to be positive, i.e. $\beta_{b}>0$, if we want to have a minimum at large volume, i.e. $x(\mathcal{V}) \geq 2 \pi$. Evaluating the second derivative at these points one finds:

$$
\frac{\partial^{2} V}{\partial \mathcal{V}^{2}}>0 \quad \Leftrightarrow \quad 4 x^{2}-15 x+9<0 .
$$

Hence the scalar potential has a minimum only for:

$$
\frac{3}{4}<x(\mathcal{V})<3
$$

provided one can find values of $\lambda_{0}$ that satisfy (4.22) for this range of values for $\mathcal{V}$. However these minima are not trustworthy since the blow-up mode $t_{s}$ is fixed below the string scale as $\left\langle t_{s}\right\rangle \simeq x(\mathcal{V}) /(2 \pi)<3 /(2 \pi)$. Moreover, the above derivation assumed a regime $x(\mathcal{V})>2 \pi$ but leads to a condition $x(\mathcal{V})<3$ for a minimum to exist, demonstrating the absence of a minimum for the $T$-moduli in a controlled region of the scalar potential. This is consistent with a numerical analysis of the scalar potential (4.18) which shows that in the range $3 / 4<x<3$ the only critical point is a saddle point with one tachyonic direction.

\subsection{Inclusion of $\alpha^{\prime}$ effects}

Let us now try to improve this situation taking into account also $\alpha^{\prime}$ corrections to the Kähler potential described in section 2.2.1. Including both $\mathcal{O}\left(\alpha^{\prime 2}\right)$ and $\mathcal{O}\left(\alpha^{\prime 3}\right)$ effects, the Kähler potential for the $T$-moduli receives the following corrections:

$$
K \simeq-\ln \mathcal{V}+\frac{\left|c_{b}\right|}{\mathcal{V}^{2 / 3}}-\frac{\gamma_{s} t_{s}+\xi / 2}{\mathcal{V}}, \quad \text { with } \quad \gamma_{s} \equiv\left|c_{b}\right| k_{s}^{1 / 3}-|\kappa| k_{b}^{1 / 3},
$$

where we have used eq. (2.21) for the expression for $c_{s}$. These higher-derivative corrections break the no-scale structure as (neglecting threshold effects):

$$
\sum_{T} K^{i \bar{j}} K_{i} K_{\bar{j}}-3 \simeq-\frac{2\left|c_{b}\right|}{\mathcal{V}^{2 / 3}}+\frac{2 \gamma_{s} t_{s}+3 \xi}{\mathcal{V}}
$$

The scalar potential (4.18) gets modified and reads:

$$
V=\left[\frac{A_{1}}{\mathcal{V}^{2 / 3}}-\frac{\left|c_{b}\right|}{\mathcal{V}^{5 / 3}}-\frac{\left|A_{2}\right|}{\left|W_{0}^{\text {eff }}\right|} \frac{t_{s}}{\mathcal{V}} e^{-\mu t_{s}}+\frac{\left|A_{3}\right|}{\left|W_{0}^{\text {eff }}\right|^{2}} \frac{e^{-2 \mu t_{s}}}{t_{s}}+\frac{\gamma_{s} t_{s}+3 \xi / 2}{\mathcal{V}^{2}}\right] \frac{\left|W_{0}^{\text {eff }}\right|^{2}}{\langle\phi\rangle}
$$


Minimising with respect to $t_{s}$ we find:

$$
\begin{aligned}
\mathcal{V} & =\left(1 \pm \sqrt{1+\frac{4\left|A_{3}\right| \gamma_{s}\left(2 \mu t_{s}+1\right)}{\left|A_{2}\right|^{2} t_{s}^{2}\left(\mu t_{s}-1\right)^{2}}}\right) \frac{\left|A_{2}\right|\left|W_{0}^{\text {eff }}\right| t_{s}^{2}\left(\mu t_{s}-1\right)}{2\left|A_{3}\right|\left(2 \mu t_{s}+1\right)} e^{\mu t_{s}} \\
& \underset{\mu t_{s} \gg 1}{\simeq} \frac{\left|A_{2}\right|\left|W_{0}^{\text {eff }}\right|}{4\left|A_{3}\right|}\left(1+\sqrt{1+\frac{c}{t_{s}^{3}}}\right) t_{s}^{2} e^{\mu t_{s}} \quad \text { with } \quad c=\frac{8\left|A_{3}\right| \gamma_{s}}{\left|A_{2}\right|^{2} \mu}=\frac{2 \gamma_{s}}{3 k_{s} \mu},
\end{aligned}
$$

where we focused only on the solution which for $\gamma_{s}=0$ correctly reduces to (4.19) since the other solution can be shown to give rise to a maximum along the $t_{s}$ direction. Note that we did not take an expansion for small $c / t_{s}^{3}$ even if this quantity is suppressed by $\mu t_{s} \gg 1$ since a large denominator might be compensated by a large value of the unknown coefficient $\gamma_{s}$. Performing the following approximation:

$$
\mu t_{s}=\ln \left(\frac{\mathcal{V}}{|\lambda|}\right)-2 \ln t_{s} \underset{t_{s} \sim \mathcal{O}(1)}{\simeq} \ln \left(\frac{\mathcal{V}}{|\lambda|}\right) \equiv x(\mathcal{V}),
$$

with:

$$
|\lambda| \equiv \frac{\left|\lambda_{0}\right|}{2}\left(1+\sqrt{1+\frac{c}{t_{s}^{3}}}\right),
$$

and substituting (4.28) in (4.27) we end up with (in the regime $x(\mathcal{V}) \gg 1$ ):

$$
V \simeq\left[A_{1} \mathcal{V}^{4 / 3}-\left|c_{b}\right| \mathcal{V}^{1 / 3}(1-\delta x)-|C| x^{3}+\frac{3 \xi}{2}\right] \frac{\left|W_{0}^{\mathrm{eff}}\right|^{2}}{\langle\phi\rangle \mathcal{V}^{2}}
$$

where we have defined:

$$
|C| \equiv \frac{\left|C_{0}\right|}{2}\left(1+\sqrt{1+\frac{c \mu^{3}}{x^{3}}}\right), \quad \text { and } \quad \delta \equiv \frac{\gamma_{s}}{\mu\left|c_{b}\right| \mathcal{V}^{1 / 3}} .
$$

Note that if we switch off the $\alpha^{\prime}$ corrections by setting $\left|c_{b}\right|=c=\xi=0$, the scalar potential (4.30) correctly reduces to (4.21) since $|\lambda| \rightarrow\left|\lambda_{0}\right|$ and $|C| \rightarrow\left|C_{0}\right|$.

Before trying to minimise this scalar potential, let us show two important facts:

- The quantity $\delta x$ is always smaller than unity since from (4.25) one finds that:

$$
\gamma_{s} \leq\left|c_{b}\right| k_{s}^{1 / 3} \quad \Rightarrow \quad \delta x \leq \frac{k_{s}^{1 / 3} t_{s}}{\mathcal{V}^{1 / 3}} \simeq \epsilon_{s}^{1 / 3} \ll 1 .
$$

Therefore the term in (4.30) proportional to $\left|c_{b}\right|$ has always a positive sign.

- If the condition $|c| / t_{s}^{3} \ll 1$ is not satisfied, there is no minimum for realistic values of the underlying parameters. In fact, in this case the term proportional to $|C|$ is always sub-leading with respect to the term proportional to $c_{b}$ since for $c \geq 0$ :

$$
R \equiv \frac{|C| x^{3}}{\left|c_{b}\right| \mathcal{V}^{1 / 3}} \leq\left(1+\sqrt{1+\frac{c}{t_{s}^{3}}}\right) \frac{t_{s}^{3}}{c} \frac{\epsilon_{s}^{1 / 3}}{x} \ll\left(1+\sqrt{1+\frac{c}{t_{s}^{3}}}\right) \frac{t_{s}^{3}}{c},
$$

which for $c / t_{s}^{3} \sim \mathcal{O}(1)$ reduces to $R \ll \mathcal{O}(1)$, whereas for $c / t_{s}^{3} \gg 1$ reduces to $R \ll \sqrt{t_{s}^{3} / c} \ll 1$. On the other hand, for $c<0$, one has $|c| / t_{s}^{3} \leq 1$ but if $|c| / t_{s}^{3} \sim \mathcal{O}(1)$, 
the ratio $R$ can be shown to reduce again to $R<\epsilon_{s}^{1 / 3} / x \ll 1$. Therefore in this case the leading order scalar potential is given by (neglecting the term proportional to $\delta$ ):

$$
V \simeq\left[A_{1} \mathcal{V}^{4 / 3}-\left|c_{b}\right| \mathcal{V}^{1 / 3}+\frac{3 \xi}{2}\right] \frac{\left|W_{0}^{\mathrm{eff}}\right|^{2}}{\langle\phi\rangle \mathcal{V}^{2}}
$$

with:

$$
|c| \gtrsim t_{s}^{3} \quad \Rightarrow \quad\left|c_{b}\right| \geq \frac{\gamma_{s}}{k_{s}^{1 / 3}}=\frac{3 k_{s}^{2 / 3} \mu}{2} c \gtrsim \frac{3 k_{s}^{2 / 3} \mu}{2} t_{s}^{3} .
$$

However the potential (4.34) has a minimum only if:

$$
\xi>\frac{5}{12}\left|c_{b}\right| \mathcal{V}^{1 / 3} \gtrsim \frac{5}{8} k_{s} t_{s}^{3} \frac{x}{\epsilon_{s}^{1 / 3}} \gg 1
$$

which is never the case for ordinary $\mathrm{CY}$ three-folds with $\xi \sim \mathcal{O}(1)$. As an illustrative example, for $\mathcal{V}=20, t_{s}=1.5$ and $k_{s}=n / 6$ with $n \in \mathbb{N}$, one finds $\xi \gtrsim 11 n^{2 / 3} \geq$ 11, corresponding to a CY with Euler number negative and very large in absolute value: $|\chi|=2\left(h^{1,2}-h^{1,1}\right) \gtrsim 4548$, while most of the known CY manifolds have $|\chi| \lesssim \mathcal{O}(1000)$

Hence we have shown that in order to have a trustable minimum we need to be in a region where $|c| \ll t_{s}^{3}$. In this case, the scalar potential (4.30) simplifies to:

$$
V \simeq\left[A_{1} \mathcal{V}^{4 / 3}-\left|c_{b}\right| \mathcal{V}^{1 / 3}(1-\delta x)-\left|C_{0}\right| x^{3}+\frac{3 \xi}{2}\right] \frac{\left|W_{0}^{\mathrm{eff}}\right|^{2}}{\langle\phi\rangle \mathcal{V}^{2}},
$$

where we have approximated $|C| \simeq\left|C_{0}\right|$. Note that the sign of the numerical coefficient $A_{1}$ is a priori undefined and depends on the sign of the underlying parameter $\beta_{b}$.

The new extrema of $V$ are located at:

$$
A_{1} \mathcal{V}^{4 / 3}=3\left|C_{0}\right| x^{2}\left(x-\frac{3}{2}\right)+\frac{5\left|c_{b}\right|}{2} \mathcal{V}^{1 / 3}\left(1-\frac{6 \delta x}{5}+\frac{3 \delta}{5}\right)-\frac{9 \xi}{2}
$$

and the second derivative at these points is positive if:

$$
u(x) \equiv 12 \xi-5\left|c_{b}\right| \mathcal{V}^{1 / 3}\left(1-\frac{8 \delta x}{5}+2 \delta\right)-2\left|C_{0}\right| x\left(4 x^{2}-15 x+9\right)>0 .
$$

Note that for $\left|c_{b}\right|=\delta=\xi=0$ (4.38) and (4.39) correctly reduce to (4.22) and (4.23) respectively. However we shall now show that by including $\alpha^{\prime}$ corrections we can find a vacuum with $x \gg 1$ where we can trust the effective field theory.

The value of the vacuum energy is:

$$
\langle V\rangle=\frac{\left|W_{0}^{\mathrm{eff}}\right|^{2}}{2\langle\phi\rangle \mathcal{V}^{2}} v(x),
$$

where:

$$
v(x) \equiv-6 \xi+\left|C_{0}\right| x^{2}(4 x-9)+3\left|c_{b}\right| \mathcal{V}^{1 / 3}\left(1-\frac{4 \delta x}{3}+\delta\right)
$$


Let us perform the following tuning to get a Minkowski vacuum:

$$
v(x)=0 \quad \text { if } \quad 6 \xi=3\left|c_{b}\right| \mathcal{V}^{1 / 3}\left(1-\frac{4 \delta x}{3}+\delta\right)+\left|C_{0}\right| x^{2}(4 x-9),
$$

and substitute it in (4.39) obtaining:

$$
u(x) \equiv\left|c_{b}\right| \mathcal{V}^{1 / 3}(1-4 \delta)+12\left|C_{0}\right| x\left(x-\frac{3}{2}\right)>0,
$$

which is automatically satisfied for $\delta \ll 1$ and $x \gg 1$. Substituting (4.42) also in the vanishing of the first derivative (4.38), this simplifies to:

$$
4 A_{1} \mathcal{V}^{4 / 3}=\left|c_{b}\right| \mathcal{V}^{1 / 3}(1-3 \delta)+9\left|C_{0}\right| x^{2},
$$

showing that if we want to have a Minkowski minimum $A_{1}$ has to be positive, i.e. $\beta_{b}>0$.

\subsection{Minkowski solutions}

Let us first define our use of the term 'Minkowski solutions'. Owing to the lack of tuning freedom in the heterotic three-form flux superpotential, achieving vacua with exponentially small vacuum energy is a real challenge. Thus we shall use the terminology 'Minkowski vacuum' to refer to a vacuum with a cosmological constant suppressed by at least a 1-loop factor $1 /\left(8 \pi^{2}\right) \simeq 0.01$ compared to the height of the barrier in the scalar potential (of order $\left.m_{3 / 2}^{2} M_{P}^{2}\right)$ which protects the $T$-moduli from run-away.

The solutions depend on seven underlying parameters: $k_{s}, k_{b}, \beta_{b},|B|,\left|c_{b}\right|,|\kappa|$ and $\xi$. We do not consider $\left|W_{0}^{\text {eff }}\right|$ as a free variable at this stage since we fix its value at $\left|W_{0}^{\text {eff }}\right|=0.06$ by the phenomenological requirement of obtaining the right GUT coupling corresponding to $\langle s\rangle \simeq\langle\phi\rangle \simeq 2$. Let us now describe a strategy to find the values of these underlying parameters which give Minkowski vacua for desired values of the moduli and within the regime of validity of all our approximations.

1. Choose the desired values for $\mathcal{V}$ and $t_{s}$ (so fixing the value of $x=2 \pi t_{s}$ ). Then work out the value of $|B|$ as a function of $k_{s}$ from (4.20).

2. Choose the desired value of $t_{b}$ and work out the value of $k_{b}$ as a function of $k_{s}$ from (4.1).

3. Determine $\left|c_{b}\right|$ as a function of $k_{s}, \xi$ and $|\kappa|$ from (4.42).

4. Derive the value of $\beta_{b}$ as a function of $k_{s}, \xi$ and $|\kappa|$ from (4.44).

5. Choose the values of $k_{s}, \xi$ and $|\kappa|$ so that all our approximations are under control, i.e. $\epsilon_{\phi}$ defined in (4.8) satisfies $\epsilon_{\phi} \ll 1, \epsilon_{s}$ defined in (4.9) gives $\epsilon_{s} \ll 1, \delta$ defined in (4.31) satisfies $\delta \ll 1$ and $\epsilon_{\alpha^{\prime}} \equiv \xi /(2 \mathcal{V}) \ll 1$. These values of $k_{s}, \xi$ and $|\kappa|$ then give the values of $k_{b},|B|,\left|c_{b}\right|$ and $\beta_{b}$ knowing that this Minkowski vacuum is fully consistent. 


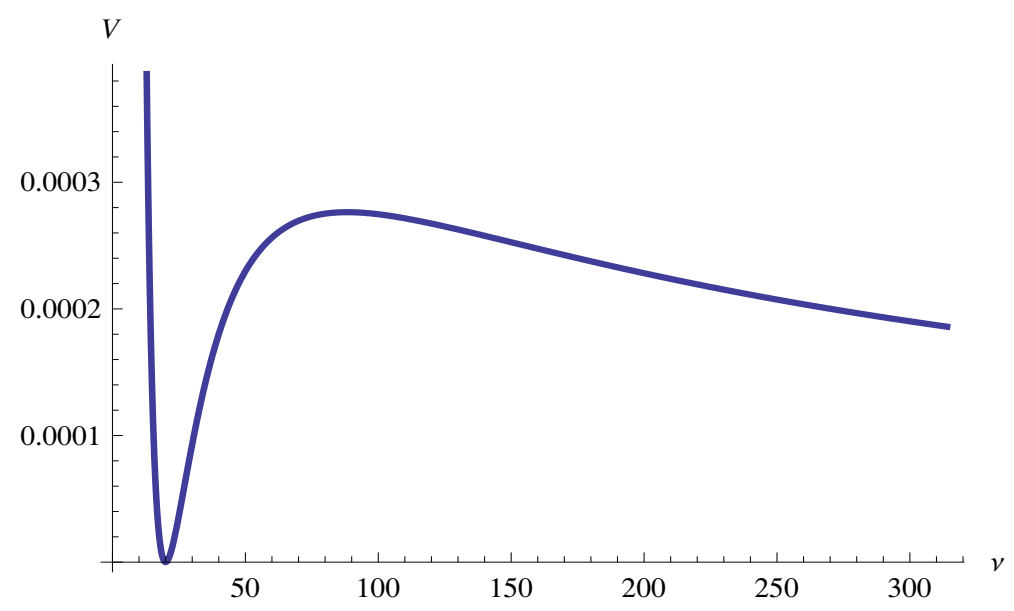

Figure 2. $V$ versus $\mathcal{V}$ assuming the parameters listed in the text which give rise to a near-Minkowski vacuum with $\langle\mathcal{V}\rangle=20$ and a cosmological constant of small magnitude compared to height of the barrier set by $m_{3 / 2}^{2} M_{P}^{2}$.

As an illustrative example, following this procedure we found a Minkowski vacuum (see figure 2) located at:

$$
\langle\phi\rangle \simeq\langle s\rangle=2, \quad\langle\mathcal{V}\rangle=20, \quad\left\langle t_{b}\right\rangle \simeq 5, \quad\left\langle t_{s}\right\rangle=1.5
$$

for the following choice of the microscopic parameters:

$$
\begin{aligned}
& k_{b}=k_{s}=1 / 6, \quad \beta_{b} \simeq 0.035, \quad\left|W_{0}^{\mathrm{eff}}\right|=0.06, \quad c_{b}=0.75, \quad c_{s}=-0.75 \\
& B \simeq 3, \quad \xi \simeq 1.49, \quad \mu=2 \pi \quad \Rightarrow \quad \gamma_{s} \simeq 0.41, \quad \kappa=0 .
\end{aligned}
$$

Note that one can get dS or AdS solutions by varying $\beta_{b}$ either above or below its benchmark value. Moreover, our approximations are under control since:

$$
\epsilon_{\phi} \simeq 0.043, \quad \epsilon_{s} \simeq 0.027, \quad \epsilon_{\alpha^{\prime}} \simeq 0.037, \quad \delta \simeq 0.032 .
$$

We stress that at the minimum these four quantities are all of the same size: $\epsilon_{\phi} \simeq \epsilon_{s} \simeq$ $\epsilon_{\alpha^{\prime}} \simeq \delta$. This has to be the case since they weight the relative strengths of loops, nonperturbative and higher derivative effects which all compete to give a minimum.

Moreover, we point out that there seem to be problems with the $\alpha^{\prime}$ expansion since we managed to obtain a minimum by tuning the underlying parameters in order to have the $\mathcal{O}\left(\alpha^{\prime 2}\right)$ term of the same order of magnitude of the $\mathcal{O}\left(\alpha^{\prime 3}\right)$ term, and so higher order $\alpha^{\prime}$ corrections might not be negligible.

However this might not be a problem if at least one of the following is valid:

- The $\mathcal{O}\left(\alpha^{\prime 2}\right)$ corrections could be eliminated by a proper redefinition of the moduli.

- The coefficients of higher order $\alpha^{\prime}$ corrections are not tuned larger than unity, resulting in a $\alpha^{\prime}$ expansion which is under control. In fact, the $\alpha^{\prime}$ expansion parameter 
is of order $q \mathcal{V}^{-1 / 3}$ with $q$ an unknown coefficient. Thus $\mathcal{O}\left(\alpha^{4}\right)$ contributions to the scalar potential can be estimated as:

$$
\frac{V_{\alpha^{\prime 4}}}{V_{\alpha^{\prime 3}}} \simeq \frac{2 q}{3 \xi \mathcal{V}^{1 / 3}} \simeq 0.16 \quad \text { for } \quad q=1
$$

\subsection{D-term potential}

So far only F-terms have been taken into account. This could be consistent since modulidependent D-terms might not be present in the absence of anomalous $\mathrm{U}(1) \mathrm{s}$, or they might be cancelled by giving suitable VEVs to charged matter fields.

However let us see how D-terms might change the previous picture in the presence of anomalous U(1)s but without introducing charged matter fields. Because of the U(1)invariance of the superpotential (4.5), both $\Phi$ and $T_{s}$ have to be neutral. Therefore the only field which can be charged under an anomalous $\mathrm{U}(1)$ is $T_{b}$ with $q_{T_{b}}=4 c_{1}^{b}(\mathcal{L}) \neq 0$. From (2.31), this induces an FI-term of the form:

$$
\xi=-q_{T_{b}} K_{b}=q_{T_{b}}\left(\frac{k_{b}}{\mathcal{V}}\right)^{1 / 3}
$$

which gives the following D-term potential:

$$
V_{D}=\frac{\xi^{2}}{\operatorname{Re}(f)} \simeq \frac{p}{\mathcal{V}^{2 / 3}} \quad \text { with } \quad p \equiv \frac{q_{T_{b}}^{2} k_{b}^{2 / 3}}{\phi} .
$$

This term has the same volume scaling as the first term in (4.37) which is the contribution coming from threshold effects. However the ratio between these two terms scales as:

$$
\frac{V_{\text {threshold }}}{V_{D}}=\frac{\epsilon_{\phi}}{\mathcal{V}^{1 / 3}} \frac{\left|W_{0}^{\text {eff }}\right|^{2}}{q_{T_{b}}^{2} k_{b}^{2 / 3}} \ll 1,
$$

for $\epsilon_{\phi} \ll 1$ and $q_{T_{b}} \sim \mathcal{O}(1)$. As an illustrative example, our explicit parameter choice would give $V_{\text {threshold }} / V_{D} \simeq 2 \cdot 10^{-4} q_{T_{b}}^{-2}$, showing that $V_{D}$ is always dominant with respect to the F-term potential (4.37). In this case, $V_{D}$ would give a run-away for the volume direction and destroy our moduli stabilisation scenario.

As we have already pointed out, this might not be the case if there are no anomalous $\mathrm{U}(1) \mathrm{s}$ or if the FI-term is cancelled by a matter field VEV. There is however another wayout to this D-term problem which relies on the possibility to fix all the moduli charged under anomalous U(1)s in a completely supersymmetric way, so ensuring the vanishing of the D-term potential. This requires $q_{T_{b}}=0$ and the addition of a third Kähler modulus $T_{c}$ which is charged under an anomalous $\mathrm{U}(1): q_{T_{c}} \neq 0$. Let us describe this situation in the next subsection.

\subsubsection{D + F-term stabilisation}

The Kähler and superpotential now read:

$$
\begin{aligned}
W & =W_{\text {flux }}(Z)+A e^{-\lambda\left(\Phi-\frac{\beta_{c}}{2} T_{c}\right)}+B e^{-\mu T_{s}}, \\
K & =-\ln \tilde{\mathcal{V}}-\ln \left[\Phi+\bar{\Phi}+\frac{\beta_{b}}{2}\left(T_{b}+\bar{T}_{b}\right)\right]+K_{\mathrm{cs}}(Z),
\end{aligned}
$$


with

$$
\tilde{\mathcal{V}}=\mathcal{V}-k_{c} t_{c}^{3}=k_{b} t_{b}^{3}-k_{s} t_{s}^{3}-k_{c} t_{c}^{3} .
$$

Note that now $\Phi$ has to get charged under an anomalous U(1) so that the hidden sector gauge kinetic function $f_{\text {hid }}=\Phi-\frac{\beta_{c}}{2} T_{c}$ becomes gauge invariant. In particular we will have $q_{\Phi}=\frac{\beta_{c}}{2} q_{T_{c}}$. From (2.31), the FI-term looks like:

$$
\xi=-q_{\Phi} \frac{D_{\Phi} W}{W}-q_{T_{c}} \frac{D_{T_{c}} W}{W},
$$

implying that $V_{D}=0$ if both $\Phi$ and $T_{c}$ are fixed supersymmetrically. However we have already seen that if all the Kähler moduli are fixed supersymmetrically via threshold effects, then perturbation theory breaks down in the hidden sector and the visible sector gauge kinetic function becomes negative. A way-out proposed in section 3.2.2 was to include worldsheet instantons but, given that we want to break supersymmetry at leading order along $T_{b}$ and $T_{s}$, in order to follow this possibility we should include a fourth modulus with worldsheet instantons. Thus this case does not look very appealing since it requires at least four moduli.

A simpler solution can be found by noticing that the problems with $\operatorname{Re}\left(f_{\text {hid }}^{1-\text { loop }}\right)>$ $\operatorname{Re}\left(f_{\text {hid }}^{\text {tree }}\right)$ and $\operatorname{Re}\left(f_{\text {vis }}\right)<0$ could be avoided if only some but not all of the Kähler moduli are fixed supersymmetrically by threshold effects. We shall now prove that this is indeed the case if the $T$-moduli fixed in this way are blow-up modes like $t_{c}$. In fact, the solution to $D_{T_{c}} W=0$ gives:

$$
\beta_{c}=-\frac{s}{\mathcal{V}} k_{c j k} t_{j} t_{k}=-\frac{6 s}{\mathcal{V}} k_{c} t_{c}^{2} .
$$

This result, in turn, gives hidden and visible sector gauge kinetic functions of the form:

$$
\frac{\operatorname{Re}\left(f_{\text {hid }}^{1-\text { loop }}\right)}{\operatorname{Re}\left(f_{\text {hid }}^{\text {tree }}\right)}=-\frac{\beta_{b} t_{b}}{2 s}-\frac{\beta_{c} t_{c}}{2 s}=-\epsilon_{\phi}+3 \frac{k_{c} t_{c}^{3}}{\mathcal{V}}=-\epsilon_{\phi}+3 \epsilon_{s} \ll 1,
$$

and:

$$
\operatorname{Re}\left(f_{\mathrm{vis}}\right)=s\left(1+\frac{\beta_{b} t_{b}}{2 s}+\frac{\beta_{c} t_{c}}{2 c}\right)=s\left(1+\epsilon_{\phi}-3 \frac{k_{c} t_{c}^{3}}{\mathcal{V}}\right)=s\left(1+\epsilon_{\phi}-3 \epsilon_{s}\right) \simeq s>0 .
$$

\section{Moduli mass spectrum, supersymmetry breaking and soft terms}

Expanding the effective field theory around the vacua found in the previous section, we can derive the moduli mass spectrum which turns out to be (see (4.29) and (4.31) for the definitions of $x$ and $\delta$ ):

$$
\begin{aligned}
m_{t_{s}} & \simeq m_{a_{s}} \simeq m_{3 / 2} x, \\
m_{Z^{\alpha}} & \simeq m_{\Phi} \simeq m_{3 / 2}, \\
m_{t_{b}} & \simeq m_{3 / 2} \delta \\
m_{a_{b}} & \simeq 0
\end{aligned}
$$


Note that in the absence of $T_{b}$-dependent worldsheet instantons which would give $a_{b}$ a mass of order $m_{a_{b}} \simeq M_{P} e^{-\mu t_{b}} \simeq 10 \mathrm{TeV}$ for $t_{b} \simeq 5$, this axion might be a good QCD axion candidate since it could remain a flat direction until standard QCD non-perturbative effects give it a tiny mass.

Moreover, the stabilisation procedure described in the previous sections leads to vacua which break supersymmetry spontaneously mainly along the Kähler moduli directions. In fact, from the general expression of the F-terms and the gravitino mass:

$$
F^{i}=e^{K / 2} K^{i \bar{j}} D_{\bar{j}} \bar{W} \quad \text { and } \quad m_{3 / 2}=e^{K / 2}|W| \simeq \frac{\left|W_{0}^{\text {eff }}\right|}{\mathcal{V}^{1 / 2}}
$$

we find that the Kähler moduli F-terms read:

$$
\frac{F^{T_{b}}}{t_{b}}=-2 m_{3 / 2} \quad \text { and } \quad \frac{F^{T_{s}}}{t_{s}} \simeq \frac{m_{3 / 2}}{x} .
$$

On the other hand, the dilaton and the complex structure moduli are fixed supersymmetrically at leading order. However, due to the fact that the prefactor of worldsheet instantons and $\alpha^{\prime}$ effects are expected to depend on these moduli, they would also break supersymmetry at sub-leading order developing F-terms whose magnitude can be estimated as: ${ }^{14}$

$$
D_{Z^{\alpha}, \Phi} W \simeq D_{Z^{\alpha}, \Phi} W_{\mathrm{wi}} \simeq \delta\left|W_{0}^{\mathrm{eff}}\right| \quad \Rightarrow \quad F^{Z^{\alpha}, \Phi} \simeq \delta m_{3 / 2} .
$$

Thus we can see that supersymmetry is mainly broken along the $t_{b}$-direction since:

$$
\frac{F^{T_{b}}}{m_{3 / 2}} \simeq t_{b} \gg \frac{F^{T_{s}}}{m_{3 / 2}} \simeq \frac{t_{s}}{x} \gg \frac{F^{Z^{\alpha}, \Phi}}{m_{3 / 2}} \simeq \delta
$$

The goldstino is therefore mainly the $T_{b}$-modulino which is eaten up by the gravitino in the super-Higgs mechanism.

Soft supersymmetry breaking terms are generated in the visible sector via tree-level gravitational interactions due to moduli mediation. Let us now derive their expressions:

- Gaugino masses: Their canonically normalised expression is given by:

$$
M_{1 / 2}=\frac{1}{2 \operatorname{Re}\left(f_{\mathrm{vis}}\right)} F^{i} \partial_{i} f_{\mathrm{vis}} \simeq \frac{F^{\Phi}}{2 \phi}+\delta \frac{F^{T_{b}}}{t_{b}} \simeq \delta m_{3 / 2}
$$

showing that the gaugino masses are suppressed with respect to the gravitino mass by a factor of order $\delta \simeq 0.03$.

- Scalar masses: The canonically normalised scalar masses generated by gravity mediation read:

$$
m_{0, \alpha}^{2}=m_{3 / 2}^{2}-F^{i} \bar{F}^{\bar{j}} \partial_{i} \partial_{\bar{j}} \ln \tilde{K}_{\alpha},
$$

where $\tilde{K}_{\alpha}$ is the Kähler metric for matter fields which we assumed to be diagonal. $\tilde{K}_{\alpha}$ is generically a function of all the moduli but we shall neglect its dependence on the dilaton and the complex structure moduli since they give only a sub-leading

\footnotetext{
${ }^{14}$ Assuming that there are no cancellations from shifts of the minimum due to sub-leading corrections.
} 
contribution to supersymmetry breaking. Hence we shall consider a Kähler metric for matter fields of the form $\tilde{K}_{\alpha} \simeq t_{s}^{-n_{s}} t_{b}^{-n_{b}}$, where $n_{s}$ and $n_{b}$ are the so-called modular weights. In the type IIB set-up, it is possible to determine the value of $n_{b}$ by requiring physical Yukawa couplings which do not depend on the large cycle due to the localisation of the visible sector on one of the small cycles [58]. However, in the heterotic framework the situation is different. For instance, in CY compactifications close to the orbifold point the visible sector typically is constructed from split multiplets which partially live in the bulk and partially arise as twisted sector states localised at orbifold fixed points. The value of the modular weights for the different matter fields is then determined by the requirements of modular invariance. Hence, they cannot be constrained by using an argument similar to the one in [58]. We shall therefore leave them as undetermined parameters. The scalar masses turn out to be:

$$
m_{0}^{2}=m_{3 / 2}^{2}\left(1-n_{b}-\frac{n_{s}}{4 x^{2}}\right)
$$

showing that for $x \gg 1$, the modular weight $n_{b}$ has to be $n_{b} \leq 1$ in order to avoid tachyonic squarks and sleptons. If $n_{b}=1$, one has a leading order cancellation in the scalar masses which therefore get generated by the F-terms of the small cycle $t_{s}$ even if $F^{T_{s}} \ll F^{T_{b}}$ (in this case one would need $n_{s}<0$ ). This is indeed the case in type IIB models because of the no-scale structure [59]. Given that the no-scale cancellation holds in the heterotic case as well, we expect a similar cancellation to occur in our case, i.e. $n_{b}=1$, with possibly the exception of twisted matter fields at orbifold fixed points, i.e. $n_{b}<1$ for twisted states.

- A-terms: The canonically normalised A-terms look like:

$$
A_{\alpha \beta \gamma}=F^{i}\left[K_{i}+\partial_{i} \ln Y_{\alpha \beta \gamma}-\partial_{i} \ln \left(\tilde{K}_{\alpha} \tilde{K}_{\beta} \tilde{K}_{\gamma}\right)\right],
$$

where $Y_{\alpha \beta \gamma}$ are the canonically unnormalised Yukawa couplings which can in principle depend on all the moduli. Similarly to the Kähler metric for matter fields, we introduce two modular weights, $p_{b}$ and $p_{s}$, and we write the Yukawa couplings as $Y_{\alpha \beta \gamma} \simeq t_{b}^{-p_{b}} t_{s}^{-p_{s}}$. Thus the A-terms take the form:

$$
A_{\alpha \beta \gamma}=3 m_{3 / 2}\left(1+p_{b}-n_{b}+\frac{p_{s}}{2 x}-\frac{n_{s}}{2 x}-\frac{\delta}{3 x}\right) .
$$

In the type IIB case, there is again a leading order cancellation (since $n_{b}=1$ and $p_{b}=0$ given that the Yukawa couplings do not depend on the Kähler moduli due to the axionic shift-symmetry and the holomorphicity of $W$ ) which is again due to the no-scale structure [59]. Similarly to the scalar masses, we expect this leading order cancellation also in the heterotic case for matter fields living in the bulk.

- $\boldsymbol{\mu}$ and $\mathbf{B} \boldsymbol{\mu}$-term: The $\mu$-term can be generated by a standard Giudice-Masiero term in the Kähler potential $K \supset \tilde{K}\left(t_{s}, t_{b}\right) H_{u} H_{d}$ which gives again $\mu \simeq m_{3 / 2}$ and $B \mu \simeq m_{3 / 2}^{2}$. 
Summarising, we obtained a very specific pattern of soft terms with scalars heavier than the gauginos and universal A-terms and $\mu / B \mu$-term of the order the gravitino mass:

$$
m_{0} \simeq A_{\alpha \beta \gamma} \simeq \mu \simeq B \simeq m_{3 / 2} \gg M_{1 / 2} \simeq \delta m_{3 / 2}
$$

The soft masses scale with $m_{3 / 2}$ and do not depend on the mechanism which stabilises the complex structure and bundle moduli. Hence one can obtain $\mathrm{TeV}$-scale supersymmetry by considering either smooth CY models where all the complex structure moduli are fixed by the holomorphicity of the gauge bundle or orbifold constructions with a small number of untwisted $Z$-moduli (or better with no untwisted $Z$-moduli at all as in the case of some non-Abelian orbifolds). On general Calabi-Yau manifolds, we expect the soft mass scale to be of order $m_{3 / 2} \sim M_{\mathrm{GUT}}$ due the fact that in the heterotic string there is not enough freedom to tune the flux superpotential below values of $\mathcal{O}(0.1-0.01)$.

\section{Anisotropic solutions}

In this section we shall show how to generalise the previous results to obtain anisotropic compactifications with 2 large and 4 small extra dimensions which allow for a right value of the GUT scale. ${ }^{15}$ For this purpose, we shall focus on CY three-folds whose volume is [63]:

$$
\mathcal{V}=k_{b} t_{b} t_{f}^{2}-k_{s} t_{s}^{3}
$$

This CY admits a 4D K3 or $T^{4}$ divisor of volume $t_{f}^{2}$ fibered over a $2 \mathrm{D} \mathbb{P}^{1}$ base of volume $t_{b}$ with an additional del Pezzo divisor of size $t_{s}^{2}$. We shall now show how to fix the moduli dynamically in the anisotropic region $t_{b} \gg t_{f} \sim t_{s}$. We shall consider a hidden sector gauge kinetic function of the form:

$$
f_{\text {hid }}=S-\frac{\beta_{b}}{2} T_{b}-\frac{\beta_{f}}{2} T_{f} \equiv \Phi, \quad \text { with } \quad \beta_{s}=0 .
$$

The superpotential looks exactly as the one in (4.5) whereas the Kähler potential reads:

$$
K=-\ln \mathcal{V}-\ln \left[\Phi+\bar{\Phi}+\frac{\beta_{b}}{2}\left(T_{b}+\bar{T}_{b}\right)+\frac{\beta_{f}}{2}\left(T_{f}+\bar{T}_{f}\right)\right]+K_{\mathrm{cs}}(Z)
$$

Focusing on the limit where 1-loop effects are suppressed with respect to the tree-level expression of the gauge kinetic function:

$$
\epsilon_{b} \equiv \frac{\beta_{b} t_{b}}{2 \phi} \ll 1 \quad \text { and } \quad \epsilon_{f} \equiv \frac{\beta_{f} t_{f}}{2 \phi} \ll 1,
$$

the dilaton is again fixed at leading order by requiring $D_{\Phi} W=0$. On the other hand the Kähler moduli develop a subdominant potential via non-perturbative contributions, $\alpha^{\prime}$ corrections and threshold effects which break the no-scale structure as:

$$
\sum_{T} K^{i \bar{j}} K_{i} K_{\bar{j}}-3=2\left(\epsilon_{b}+\epsilon_{f}\right)+\mathcal{O}\left(\epsilon^{2}\right) .
$$

\footnotetext{
${ }^{15}$ For anisotropic solutions in the type IIB case for the same kind of fibred CY manifolds see [60-62].
} 
The scalar potential has therefore the same expression as (4.16) but with a different coefficient $A_{1}$ which is now moduli-dependent and looks like:

$$
A_{1}\left(\mathcal{V}, t_{f}\right)=\frac{\mathcal{V}^{2 / 3}}{2\langle\phi\rangle}\left(\frac{\beta_{b}}{k_{b} t_{f}^{2}}+\frac{\beta_{f} t_{f}}{\mathcal{V}}\right),
$$

where we have traded $t_{b}$ for $\mathcal{V}$. This is the only term which depends on $t_{f}$ since the rest of the potential depends just on $\mathcal{V}$ and $t_{s}$. Hence we can fix $t_{f}$ just minimising $A_{1}\left(\mathcal{V}, t_{f}\right)$ obtaining:

$$
t_{f}=\left(\frac{2 \beta_{b}}{k_{b} \beta_{f}}\right)^{1 / 3} \mathcal{V}^{1 / 3} \quad \Leftrightarrow \quad t_{f}=\frac{2 \beta_{b}}{\beta_{f}} t_{b} .
$$

Substituting this result in (6.6) we find that $A_{1}$ becomes:

$$
A_{1}=\frac{3 \beta_{b}}{2\langle\phi\rangle k_{b}^{1 / 3}}\left(\frac{\beta_{f}}{2 \beta_{b}}\right)^{2 / 3},
$$

which is not moduli-dependent anymore and takes a form very similar to the one in (4.17). We can therefore follow the same stabilisation procedure described in the previous sections but now with the additional relation (6.7) which, allowing the moderate tuning $\beta_{f} \simeq 20 \beta_{b}$, would give an anisotropic solution with $t_{b} \simeq 10 t_{f}$. For example for $\mathcal{V} \simeq 20$ and $k_{b}=1 / 2$, one would obtain $t_{b} \simeq 16 \gg t_{f} \simeq 1.6$.

We finally mention that this kind of fibred CY manifolds have been successfully used in type IIB for deriving inflationary models from string theory where the inflaton is the Kähler modulus controlling the volume of the fibre [64]. It would be very interesting to investigate if similar cosmological applications could also be present in the heterotic case.

\section{Conclusions}

The heterotic string on a CY manifold (or its various limiting cases such as orbifolds and Gepner points) has been studied since the late eighties as a possible UV complete theory of gravity that can realise a unified version of the SM. In the last decade there has been much progress towards the goal of getting a realistic model with the correct spectrum. However the major problem in getting phenomenologically viable solutions for the heterotic string is that the gauge theory resides in the bulk, and so getting an acceptable model cannot be decoupled from the problem of moduli stabilisation. Unfortunately a complete and deep understanding of the mechanism which stabilises all the moduli in the heterotic string is still lacking.

In this paper we tried to perform a systematic analysis of all the effects which can develop a potential for the various moduli for the case of $(0,2)$-compactifications which allow for MSSM-like model building and the generation of worldsheet instantons that are crucial effects to fix the Kähler moduli. According to the original Strominger's analysis [20], these compactifications violate the Kähler condition $d J=0$ due to a non-zero $H$-flux at $\mathcal{O}\left(\alpha^{\prime}\right)$ since in the non-standard embedding the co-exact piece of the Chern-Simons term 
in $H$ does not cancel. We then considered solutions to the 10D equations of motion with constant dilaton and warp factor, corresponding to 'special Hermitian manifolds', which represent the smallest deviations from smooth $\mathrm{CY}$ manifolds at $\mathcal{O}\left(\alpha^{\prime}\right)[40]$.

Let us summarise the various moduli stabilisation effects that we have taken into account:

- Holomorphicity of the gauge bundle, D-terms and higher order perturbative contributions to the superpotential: By demanding a supersymmetric gauge bundle, i.e. a gauge bundle which satisfies the Hermitian Yang-Mills equations, the combined space of complex structure and gauge bundle moduli reduces from a naive direct product to a 'cross-structure' [33-36]. Therefore if the gauge bundle moduli are fixed at nonzero VEVs by D-terms combined with higher order perturbative contributions to the superpotential [4-8], the $Z$-moduli are automatically lifted. However, not all the complex structure moduli might get frozen by this mechanism since, in general, the sub-locus in complex structure moduli space where the gauge bundle is holomorphic turns out to have dimension $h_{\text {hol }}^{1,2}>0$. Hence $0<h_{\text {hol }}^{1,2}<h^{1,2}$ flat $Z$-moduli are generically left over.

- Fractional Chern-Simons invariants, gaugino condensation and threshold effects: The remaining flat $Z$-directions could be lifted by turning on quantised background threeform fluxes [17-19]. However we showed that, contrary to type IIB, this cannot be done having at the same time a vanishing VEV of the tree-level flux superpotential $W_{0}$ since setting the F-terms of the $Z$-moduli to zero corresponds to setting the $(1,2)$-component of the $H$-flux to zero, while demanding $W_{0}=0$ implies that also the $(3,0)$-piece of $H$ is vanishing. Hence, being real, the whole $H$-flux has to be zero, resulting in the impossibility of fixing the remaining complex structure moduli. Thus one needs $W_{0} \neq 0$ in order to fix the $Z$-moduli. However, due to the absence of Ramond-Ramond fluxes, it is hard to tune $W_{0}$ small enough to balance the exponentially suppressed contribution from gaugino condensation which introduces an explicit dependence on the dilaton [11-13] unless one turns on fractional Chern-Simons invariants (i.e. discrete Wilson lines) [17]. In this way both the dilaton and the complex structure moduli can be stabilised supersymmetrically at non-perturbative level. The Kähler moduli could then be fixed by the inclusion of threshold corrections to the gauge kinetic function $[21,22]$.

- Worldsheet instantons: The supersymmetric minimum obtained by including threshold effects is not in the weak coupling regime where one can trust the effective field theory. This problem can be avoided by considering also the contribution of $T$ dependent worldsheet instantons which can give rise to reliable supersymmetric AdS vacua [19].

- Higher derivative and loop corrections to the Kähler potential: The last effects to be taken into account are $\alpha^{\prime}$ corrections to the Kähler potential [23-25], while string loop effects can be estimated to give rise to negligible contributions to the scalar 
potential [48-50]. These higher derivative corrections yield new stable vacua where supersymmetry is spontaneously broken by the stabilisation mechanism which induces non-zero F-terms for the Kähler moduli, in a way very similar to type IIB LARGE Volume Scenarios [26, 27]. These new vacua can be Minkowski due to the positive contribution from threshold effects. However, due to the lack of tuning freedom in $W_{0}$, it is very hard to achieve vacua with exponentially small vacuum energy. Thus we used the term 'Minkowski vacua' to refer to solutions with a cosmological constant suppressed by at least a loop factor with respect to the height of the barrier in the scalar potential which prevents the Kähler moduli to run-away to infinity. Moreover, this stabilisation mechanism allows for anisotropic compactifications with two extra dimensions which are much larger than the other four. In this way, the unification scale can be lowered down to the observed phenomenological value [28, 29], fitting very well with the picture of $6 \mathrm{D}$ orbifold GUTs [29, 30].

After showing the existence of this new kind of supersymmetry breaking vacua, we estimated the size of the soft terms generated by gravity mediation. Interestingly, they feature universal scalar masses, A-terms and $\mu / B \mu$-term of $\mathcal{O}\left(m_{3 / 2}\right)$ and suppressed gaugino masses at the \%-level. Moreover, a potentially viable QCD axion candidate is given by the axionic partner of the 'large' 2-cycle modulus. However, due to the lack of tuning freedom in the flux superpotential $W_{0} \simeq \mathcal{O}(0.1-0.01)$, the gravitino mass $m_{3 / 2}=W_{0} M_{P} / \sqrt{2 \operatorname{Re}(\mathrm{S}) \mathcal{V}}$ becomes of order $M_{G U T} \simeq 10^{16} \mathrm{GeV}$ for $\operatorname{Re}(\mathrm{S}) \simeq 2$ and $\mathcal{V} \simeq 20$. This is not a problem if one does not believe in the solution of the hierarchy problem based on low-energy supersymmetry, but it represents a generic prediction of weakly coupled heterotic compactifications on internal manifolds which are smooth CY three-folds up to $\alpha^{\prime}$ effects.

However, our stabilisation procedure for the Kähler moduli that leads to spontaneous supersymmetry breaking, is completely independent on the supersymmetric mechanism which is used to fix the dilaton and the complex structure moduli. Hence, if one is instead interested in low-energy supersymmetry, our way to break supersymmetry along the Kähler moduli directions could still be used by focusing on different ways to freeze the $S$ and $Z$-moduli:

1. In some particular examples all the complex structure moduli could be stabilised by the requirement of a holomorphic gauge bundle [33-35]. In this case one could have $W_{0}=0$ and an exponentially small superpotential, leading to a $\mathrm{TeV}$-scale gravitino mass, could be generated by gaugino condensation.

2. In Abelian orbifold models the number of untwisted complex structure moduli is very small. There are also some non-Abelian orbifolds with no $Z$-moduli at all. Hence in this case it is rather likely that all the $Z$-moduli could be fixed by the holomorphicity of the gauge bundle once all the singlets are fixed at non-zero VEVs by cancelling FI-terms or by the effect of higher order terms in $W$ [4-8]. Again, $m_{3 / 2}$ could then be lowered to the $\mathrm{TeV}$-scale due to the exponential suppression coming from gaugino condensation $[31,32]$. 
3. The flux superpotential could have enough tuning freedom in the presence of fluxes which are the equivalent of type IIB Ramond-Ramond fluxes. This is the case of non-complex manifolds with new geometric fluxes where the $H$ flux gets modified to $\mathcal{H}=H+\mathrm{i} d J[40,42-45]{ }^{16}$

We finally stress that, even if these models could give low-energy supersymmetry, the possibility to tune the cosmological constant to the observed value still remains a challenge, in particular in the cases without a large flux discretuum.

\section{Acknowledgments}

We would like to thank Lara Anderson, Lilia Anguelova, Arthur Hebecker, James Gray, Shamit Kachru, Oleg Lebedev, Andre Lukas, Anshuman Maharana, Hans Peter Nilles, Francisco Pedro, Fernando Quevedo, Callum Quigley, Stuart Raby, Marco Serone, Savdeep Sethi, Patrick Vaudrevange, Roberto Zucchini and especially Roberto Valandro for useful discussions and correspondence. We are grateful for the support of, and the pleasant environs provided by, the Abdus Salam International Center for Theoretical Physics. This work was supported by the Impuls und Vernetzungsfond of the Helmholtz Association of German Research Centres under grant HZ-NG-603, and German Science Foundation (DFG) within the Collaborative Research Center (CRC) 676 "Particles, Strings and the Early Universe", and by the United States Department of Energy under grant DE-FG0291-ER-40672. SdA would like to acknowledge the award of an CRC 676 fellowship from DESY/Hamburg University and a visiting professorship at Abdus Salam ICTP.

\section{A Dimensional reduction of $10 \mathrm{D}$ heterotic action}

The 10D heterotic supergravity action in string frame for energies below the mass of the first excited string state $M_{s}=\ell_{s}^{-1}$ with $\ell_{s}=2 \pi \sqrt{\alpha^{\prime}}$ contains bosonic terms of the form:

$$
\begin{aligned}
S & \supset \frac{1}{(2 \pi)^{7} \alpha^{\prime 4}} \int d^{10} x \sqrt{-G} e^{-2 \phi}\left(\mathcal{R}-\frac{\alpha^{\prime}}{4} \operatorname{Tr} F^{2}\right) \\
& =\frac{M_{10}^{8}}{2} \int d^{10} x \sqrt{-G} e^{-2 \phi} \mathcal{R}-\frac{1}{2 g_{10}^{2}} \int d^{10} x \sqrt{-G} e^{-2 \phi} \operatorname{Tr} F^{2} .
\end{aligned}
$$

Comparing the first with the second line in (A.1), we find:

$$
M_{10}^{8}=\frac{2}{(2 \pi)^{7} \alpha^{4}}=4 \pi M_{s}^{8} \quad \text { and } \quad g_{10}^{2}=\frac{4}{\alpha^{\prime} M_{10}^{8}}=4 \pi M_{s}^{-6} .
$$

Compactifying on a $6 \mathrm{D}$ CY three-fold $X$, the $4 \mathrm{D}$ Planck scale $M_{P}$ turns out to be:

$$
M_{P}^{2}=e^{-2\langle\phi\rangle} M_{10}^{8} \operatorname{Vol}(X)=4 \pi g_{s}^{-2} \mathcal{V} M_{s}^{2},
$$

where we measured the internal volume in units of $M_{s}^{-1}$ as $\operatorname{Vol}(X)=\mathcal{V} \ell_{s}^{6}$ and we explicitly included factors of the string coupling $g_{s}=e^{\langle\phi\rangle}$. On the other hand, the $4 \mathrm{D}$ gauge coupling constant becomes:

$$
\alpha_{G U T}^{-1}=4 \pi g_{4}^{-2}=\frac{4 \pi \mathrm{Vol}(X)}{g_{10}^{2} e^{2\langle\phi\rangle}}=g_{s}^{-2} \mathcal{V}
$$

\footnotetext{
${ }^{16}$ See also [65].
} 
The tree-level expression of the gauge kinetic function $f=S$ requires $\operatorname{Re}(S)=g_{4}^{-2}$, implying the following normalisation of the definition of the dilaton field:

$$
S=\frac{1}{4 \pi}\left(e^{-2 \phi} \mathcal{V}+\mathrm{i} a\right)
$$

From (A.4), we immediately realise that there is a tension between large volume and weak coupling for the physical value $\alpha_{G U T}^{-1} \simeq 25$ :

$$
\mathcal{V}=g_{s}^{2} \alpha_{G U T}^{-1} \simeq g_{s}^{2} 25 \lesssim 25 \quad \text { for } \quad g_{s} \lesssim 1
$$

On top of this problem, isotropic compactifications cannot yield the right value of the GUT scale $M_{G U T} \simeq 2.1 \cdot 10^{16} \mathrm{GeV}$ which is given by the Kaluza-Klein scale $M_{K K}=M_{s} / \mathcal{V}^{1 / 6}$. In fact, combining (A.3) with (A.4), one finds that the string scale is fixed to be very high:

$$
M_{s}^{2}=\frac{M_{P}^{2}}{4 \pi \alpha_{G U T}^{-1}} \simeq \frac{M_{P}^{2}}{100 \pi} \simeq\left(1.35 \cdot 10^{17} \mathrm{GeV}\right)^{2}
$$

In turn, for $\mathcal{V} \lesssim 25$, the GUT scale becomes too high: $M_{G U T}=M_{K K} \gtrsim 8 \cdot 10^{16} \mathrm{GeV}$. The situation can be improved by focusing on anisotropic compactifications with $d$ large extra dimensions of size $L=x \ell_{s}$ with $x \gg 1$ and $(6-d)$ small dimensions of string size $l=\ell_{s}$. The internal volume then becomes $\operatorname{Vol}(X)=L^{d} l^{(6-d)}=x^{d} \ell_{s}^{6}=\mathcal{V} \ell_{s}^{6}$, implying that the Kaluza-Klein scale now becomes $M_{K K}=M_{s} / x=M_{s} / \mathcal{V}^{1 / d}$. Clearly, for the case $d=6$, we recover the isotropic situation. The case with $d=1$ is not very interesting since CY manifolds do not admit non-trivial Wilson lines to perform the GUT breaking. We shall therefore focus on the case $d=2$ where we get the promising result:

$$
M_{G U T}=M_{K K}=\frac{M_{s}}{\sqrt{\mathcal{V}}} \gtrsim 2.7 \cdot 10^{16} \mathrm{GeV} .
$$

Open Access. This article is distributed under the terms of the Creative Commons Attribution License which permits any use, distribution and reproduction in any medium, provided the original author(s) and source are credited.

\section{References}

[1] M. Graña, Flux compactifications in string theory: A Comprehensive review, Phys. Rept. 423 (2006) 91 [hep-th/0509003] [InSPIRE].

[2] M.R. Douglas and S. Kachru, Flux compactification, Rev. Mod. Phys. 79 (2007) 733 [hep-th/0610102] [INSPIRE].

[3] V. Braun, Y.-H. He, B.A. Ovrut and T. Pantev, A Heterotic standard model, Phys. Lett. B 618 (2005) 252 [hep-th/0501070] [INSPIRE].

[4] W. Buchmüller, K. Hamaguchi, O. Lebedev and M. Ratz, Supersymmetric standard model from the heterotic string, Phys. Rev. Lett. 96 (2006) 121602 [hep-ph/0511035] [INSPIRE].

[5] W. Buchmüller, K. Hamaguchi, O. Lebedev and M. Ratz, Supersymmetric Standard Model from the Heterotic String (II), Nucl. Phys. B 785 (2007) 149 [hep-th/0606187] [InSPIRE]. 
[6] O. Lebedev et al., A Mini-landscape of exact MSSM spectra in heterotic orbifolds, Phys. Lett. B 645 (2007) 88 [hep-th/0611095] [INSPIRE].

[7] O. Lebedev et al., The Heterotic Road to the MSSM with R parity, Phys. Rev. D 77 (2008) 046013 [arXiv:0708.2691] [InSPIRE].

[8] O. Lebedev, H.P. Nilles, S. Ramos-Sanchez, M. Ratz and P.K. Vaudrevange, Heterotic mini-landscape. (II). Completing the search for MSSM vacua in a Z(6) orbifold, Phys. Lett. B 668 (2008) 331 [arXiv:0807.4384] [INSPIRE].

[9] B. Dundee, S. Raby and A. Westphal, Moduli stabilization and SUSY breaking in heterotic orbifold string models, Phys. Rev. D 82 (2010) 126002 [arXiv:1002.1081] [INSPIRE].

[10] S.L. Parameswaran, S. Ramos-Sanchez and I. Zavala, On Moduli Stabilisation and de Sitter Vacua in MSSM Heterotic Orbifolds, JHEP 01 (2011) 071 [arXiv: 1009.3931] [INSPIRE].

[11] A. Font, L.E. Ibáñez, D. Lüst and F. Quevedo, Supersymmetry Breaking From Duality Invariant Gaugino Condensation, Phys. Lett. B 245 (1990) 401 [INSPIRE].

[12] S. Ferrara, N. Magnoli, T. Taylor and G. Veneziano, Duality and supersymmetry breaking in string theory, Phys. Lett. B 245 (1990) 409 [INSPIRE].

[13] H.P. Nilles and M. Olechowski, Gaugino Condensation and Duality Invariance, Phys. Lett. B 248 (1990) 268 [INSPIRE].

[14] J. Casas, Z. Lalak, C. Muñoz and G.G. Ross, Hierarchical Supersymmetry Breaking and Dynamical Determination of Compactification Parameters by Nonperturbative Effects, Nucl. Phys. B 347 (1990) 243 [inSPIRE].

[15] B. de Carlos, J. Casas and C. Muñoz, Supersymmetry breaking and determination of the unification gauge coupling constant in string theories, Nucl. Phys. B 399 (1993) 623 [hep-th/9204012] [INSPIRE].

[16] D. Gallego and M. Serone, Moduli Stabilization in non-Supersymmetric Minkowski Vacua with Anomalous U(1) Symmetry, JHEP 08 (2008) 025 [arXiv:0807.0190] [INSPIRE].

[17] S. Gukov, S. Kachru, X. Liu and L. McAllister, Heterotic moduli stabilization with fractional Chern-Simons invariants, Phys. Rev. D 69 (2004) 086008 [hep-th/0310159] [INSPIRE].

[18] R. Brustein and S.P. de Alwis, Moduli potentials in string compactifications with fluxes: Mapping the discretuum, Phys. Rev. D 69 (2004) 126006 [hep-th/0402088] [InSPIRE].

[19] G. Curio, A. Krause and D. Lüst, Moduli stabilization in the heterotic/ IIB discretuum, Fortsch. Phys. 54 (2006) 225 [hep-th/0502168] [INSPIRE].

[20] A. Strominger, Superstrings with Torsion, Nucl. Phys. B 274 (1986) 253 [INSPIRE].

[21] L.J. Dixon, V. Kaplunovsky and J. Louis, Moduli dependence of string loop corrections to gauge coupling constants, Nucl. Phys. B 355 (1991) 649 [INSPIRE].

[22] R. Blumenhagen, G. Honecker and T. Weigand, Loop-corrected compactifications of the heterotic string with line bundles, JHEP 06 (2005) 020 [hep-th/0504232] [INSPIRE].

[23] L. Anguelova, C. Quigley and S. Sethi, The Leading Quantum Corrections to Stringy Kähler Potentials, JHEP 10 (2010) 065 [arXiv: 1007.4793] [INSPIRE].

[24] P. Candelas, X.C. De La Ossa, P.S. Green and L. Parkes, A Pair of Calabi-Yau manifolds as an exactly soluble superconformal theory, Nucl. Phys. B 359 (1991) 21 [INSPIRE]. 
[25] K. Becker, M. Becker, M. Haack and J. Louis, Supersymmetry breaking and alpha-prime corrections to flux induced potentials, JHEP 06 (2002) 060 [hep-th/0204254] [INSPIRE].

[26] V. Balasubramanian, P. Berglund, J.P. Conlon and F. Quevedo, Systematics of moduli stabilisation in Calabi-Yau flux compactifications, JHEP 03 (2005) 007 [hep-th/0502058] [INSPIRE].

[27] M. Cicoli, J.P. Conlon and F. Quevedo, General Analysis of LARGE Volume Scenarios with String Loop Moduli Stabilisation, JHEP 10 (2008) 105 [arXiv:0805.1029] [INSPIRE].

[28] A. Hebecker and M. Trapletti, Gauge unification in highly anisotropic string compactifications, Nucl. Phys. B 713 (2005) 173 [hep-th/0411131] [INSPIRE].

[29] B. Dundee, S. Raby and A. Wingerter, Reconciling Grand Unification with Strings by Anisotropic Compactifications, Phys. Rev. D 78 (2008) 066006 [arXiv:0805.4186] [INSPIRE].

[30] W. Buchmüller, C. Lüdeling and J. Schmidt, Local SU(5) Unification from the Heterotic String, JHEP 09 (2007) 113 [arXiv:0707.1651] [INSPIRE].

[31] O. Loaiza-Brito, J. Martin, H.P. Nilles and M. Ratz, $\log (\mathrm{M}(\mathrm{Pl}) / m(3 / 2))$, AIP Conf. Proc. 805 (2006) 198 [hep-th/0509158] [INSPIRE].

[32] R. Kappl et al., Large hierarchies from approximate $R$ symmetries, Phys. Rev. Lett. 102 (2009) 121602 [arXiv:0812.2120] [INSPIRE].

[33] L.B. Anderson, J. Gray, A. Lukas and B. Ovrut, Stabilizing the Complex Structure in Heterotic Calabi-Yau Vacua, JHEP 02 (2011) 088 [arXiv: 1010.0255] [INSPIRE].

[34] L.B. Anderson, J. Gray, A. Lukas and B. Ovrut, Stabilizing All Geometric Moduli in Heterotic Calabi-Yau Vacua, Phys. Rev. D 83 (2011) 106011 [arXiv:1102.0011] [InSPIRE].

[35] L.B. Anderson, J. Gray, A. Lukas and B. Ovrut, The Atiyah Class and Complex Structure Stabilization in Heterotic Calabi-Yau Compactifications, JHEP 10 (2011) 032 [arXiv:1107.5076] [INSPIRE].

[36] E. Witten, New Issues in Manifolds of SU(3) Holonomy, Nucl. Phys. B 268 (1986) 79 [INSPIRE].

[37] R. Donagi and M. Wijnholt, Higgs Bundles and UV Completion in F-theory, arXiv:0904.1218 [INSPIRE].

[38] R. Donagi and M. Wijnholt, Model Building with F-Theory, Adv. Theor. Math. Phys. 15 (2011) 1237 [arXiv: 0802.2969] [inSPIRE].

[39] L.B. Anderson, J. Gray, A. Lukas and B. Ovrut, Vacuum Varieties, Holomorphic Bundles and Complex Structure Stabilization in Heterotic Theories, JHEP 07 (2013) 017 [arXiv: 1304.2704] [INSPIRE].

[40] G. Lopes Cardoso et al., NonKähler string backgrounds and their five torsion classes, Nucl. Phys. B 652 (2003) 5 [hep-th/0211118] [InSPIRE].

[41] S.B. Giddings, S. Kachru and J. Polchinski, Hierarchies from fluxes in string compactifications, Phys. Rev. D 66 (2002) 106006 [hep-th/0105097] [INSPIRE].

[42] G. Lopes Cardoso, G. Curio, G. Dall'Agata and D. Lüst, BPS action and superpotential for heterotic string compactifications with fluxes, JHEP 10 (2003) 004 [hep-th/0306088] [INSPIRE]. 
[43] J. Held, D. Lüst, F. Marchesano and L. Martucci, DWSB in heterotic flux compactifications, JHEP 06 (2010) 090 [arXiv: 1004.0867] [INSPIRE].

[44] K. Becker, M. Becker, K. Dasgupta and S. Prokushkin, Properties of heterotic vacua from superpotentials, Nucl. Phys. B 666 (2003) 144 [hep-th/0304001] [INSPIRE].

[45] K. Becker, M. Becker, K. Dasgupta and P.S. Green, Compactifications of heterotic theory on nonKähler complex manifolds. 1., JHEP 04 (2003) 007 [hep-th/0301161] [INSPIRE].

[46] E. Witten, Dimensional Reduction of Superstring Models, Phys. Lett. B 155 (1985) 151 [INSPIRE].

[47] S. Ferrara, C. Kounnas and M. Porrati, General Dimensional Reduction of Ten-Dimensional Supergravity and Superstring, Phys. Lett. B 181 (1986) 263 [INSPIRE].

[48] M. Berg, M. Haack and B. Körs, String loop corrections to Kähler potentials in orientifolds, JHEP 11 (2005) 030 [hep-th/0508043] [INSPIRE].

[49] M. Berg, M. Haack and E. Pajer, Jumping Through Loops: On Soft Terms from Large Volume Compactifications, JHEP 09 (2007) 031 [arXiv: 0704.0737] [INSPIRE].

[50] M. Cicoli, J.P. Conlon and F. Quevedo, Systematics of String Loop Corrections in Type IIB Calabi-Yau Flux Compactifications, JHEP 01 (2008) 052 [arXiv:0708.1873] [INSPIRE].

[51] P. Candelas and X. de la Ossa, Moduli Space of Calabi-Yau Manifolds, Nucl. Phys. B 355 (1991) 455 [inSPIRE].

[52] B.R. Greene and M. Plesser, Duality in Calabi-Yau Moduli Space, Nucl. Phys. B 338 (1990) 15 [inSPIRE].

[53] P. Candelas, A. Font, S.H. Katz and D.R. Morrison, Mirror symmetry for two parameter models. 2., Nucl. Phys. B 429 (1994) 626 [hep-th/9403187] [INSPIRE].

[54] P. Candelas, X. de la Ossa and F. Rodriguez-Villegas, Calabi-Yau manifolds over finite fields. 1., hep-th/0012233 [INSPIRE].

[55] A. Giryavets, S. Kachru, P.K. Tripathy and S.P. Trivedi, Flux compactifications on Calabi-Yau threefolds, JHEP 04 (2004) 003 [hep-th/0312104] [INSPIRE].

[56] F. Denef, M.R. Douglas and B. Florea, Building a better racetrack, JHEP 06 (2004) 034 [hep-th/0404257] [INSPIRE].

[57] L. Anguelova and C. Quigley, Quantum Corrections to Heterotic Moduli Potentials, JHEP 02 (2011) 113 [arXiv: 1007.5047] [INSPIRE].

[58] J.P. Conlon, D. Cremades and F. Quevedo, Kähler potentials of chiral matter fields for Calabi-Yau string compactifications, JHEP 01 (2007) 022 [hep-th/0609180] [INSPIRE].

[59] J.P. Conlon, S.S. AbdusSalam, F. Quevedo and K. Suruliz, Soft SUSY Breaking Terms for Chiral Matter in IIB String Compactifications, JHEP 01 (2007) 032 [hep-th/0610129] [INSPIRE].

[60] M. Cicoli, C. Burgess and F. Quevedo, Anisotropic Modulus Stabilisation: Strings at LHC Scales with Micron-sized Extra Dimensions, JHEP 10 (2011) 119 [arXiv:1105.2107] [INSPIRE].

[61] M. Cicoli, C. Mayrhofer and R. Valandro, Moduli Stabilisation for Chiral Global Models, JHEP 02 (2012) 062 [arXiv:1110.3333] [INSPIRE]. 
[62] S. Angus and J.P. Conlon, Soft Supersymmetry Breaking in Anisotropic LARGE Volume Compactifications, JHEP 03 (2013) 071 [arXiv:1211.6927] [INSPIRE].

[63] M. Cicoli, M. Kreuzer and C. Mayrhofer, Toric K3-Fibred Calabi-Yau Manifolds with del Pezzo Divisors for String Compactifications, JHEP 02 (2012) 002 [arXiv:1107.0383] [INSPIRE].

[64] M. Cicoli, C. Burgess and F. Quevedo, Fibre Inflation: Observable Gravity Waves from IIB String Compactifications, JCAP 03 (2009) 013 [arXiv:0808.0691] [InSPIRE].

[65] K. Becker and S. Sethi, Torsional Heterotic Geometries, Nucl. Phys. B 820 (2009) 1 [arXiv: 0903.3769] [INSPIRE]. 